\title{
Existence of Solutions for Nonhomogeneous A-Harmonic Equations with Variable Growth
}

\author{
Yongqiang Fu and Lifeng Guo \\ Department of Mathematics, Harbin Institute of Technology, Harbin 150001, China \\ Correspondence should be addressed to Lifeng Guo, hitglf@yahoo.com.cn
}

Received 29 February 2012; Accepted 18 June 2012

Academic Editor: Shaoyong Lai

Copyright (c) 2012 Y. Fu and L. Guo. This is an open access article distributed under the Creative Commons Attribution License, which permits unrestricted use, distribution, and reproduction in any medium, provided the original work is properly cited.

We study the following nonhomogeneous $A$-harmonic equations: $d^{*} A(x, d u(x))+B(x, u(x))=$ $0, x \in \Omega, u(x)=0, x \in \partial \Omega$, where $\Omega \subset \mathbb{R}^{n}$ is a bounded and convex Lipschitz domain, $A(x, d u(x))$ and $B(x, u(x))$ satisfy some $p(x)$-growth conditions, respectively. We obtain the existence of weak solutions for the above equations in subspace $\mathfrak{K}_{0}^{1, p(x)}\left(\Omega, \Lambda^{l-1}\right)$ of $W_{0}^{1, p(x)}\left(\Omega, \Lambda^{l-1}\right)$.

\section{Introduction}

Spaces of differential forms have been discussed in great details (see $[1,2]$ and the references therein). The theory of differential forms is an approach to multivariable calculus that is independent of coordinates and provides a better definition for integrals. Differential forms have played an important role in physical laws of thermodynamics, analytical mechanics, and physical theories, in particular Maxwell's theory, and the Yang-Mills theory, the theory of relativity, see for example [3-6].

In recent years, the study of $A$-harmonic equations for differential forms has developed rapidly. Many interesting results concerning $A$-harmonic equation have been established recently (see [7-11] and the references therein). In [12], spaces $L^{p(x)}(\Omega)$ and $W^{k, p(x)}(\Omega)$ are first introduced, and they used them to study the solutions of nonlinear Dirichlet boundary value problems with $p(x)$-growth conditions. In [13], spaces $L^{p(x)}\left(\Omega, \Lambda^{l}, \omega\right)$ and $W^{1, p(x)}\left(\Omega, \Lambda^{l}, \omega\right)$ are first introduced and used to study the weak solutions of obstacle problems of $A$-harmonic equations with variable growth for differential forms. 
Let $\Omega \subset \mathbb{R}^{n}$ be a bounded and convex Lipschitz domain. It is our purpose to study the following systems:

$$
\begin{gathered}
d^{*} A(x, d u(x))+B(x, u(x))=0, \quad x \in \Omega, \\
u(x)=0, \quad x \in \partial \Omega,
\end{gathered}
$$

where $u \in \Lambda^{l-1}(\Omega), l=1,2, \ldots, n$, and $A: \Omega \times \Lambda^{l}(\Omega) \rightarrow \Lambda^{l}(\Omega), B: \Omega \times \Lambda^{l-1}(\Omega) \rightarrow \Lambda^{l-1}(\Omega)$ satisfy the following conditions.

(H1) $A(x, \xi)$ and $B(x, \varsigma)$ are measurable with respect to $x$ for all $\xi, \varsigma$ and continuous with respect to $\xi, \varsigma$, respectively, for a.e. $x \in \Omega$.

(H2) $|A(x, \xi)|+|B(x, \varsigma)| \leq C_{1}|\xi|^{p(x)-1}+C_{2} \mid \varsigma^{p(x)-1}+G(x)$, where $G \in L^{p^{\prime}(x)}(\Omega)$ and $C_{1}, C_{2} \geq 0$ are constants.

(H3) $\langle A(x, \xi), \xi\rangle \geq a|\xi|^{p(x)}-|h(x)|$, where $a>0$ is a constant and $h \in L^{1}(\Omega)$.

(H4) $\langle B(x, \varsigma), \varsigma\rangle \geq \bar{a}|\varsigma|^{p(x)}-|\bar{h}(x)|$, where $\bar{a} \geq 0$ is a constant and $\bar{h} \in L^{1}(\Omega)$.

(H5) For a.e. $x_{0} \in \Omega$, the mapping $\xi \rightarrow A\left(x_{0}, \xi\right)$ satisfies

$$
\int_{D}\left\langle A\left(x_{0}, \xi_{0}+d v(x)\right), d v(x)\right\rangle d x \geq \gamma \int_{D}|d v(x)|^{p(x)} d x
$$

for each $\xi_{0} \in \Lambda^{l}(\Omega), D \subset \Omega$ and $v \in C_{0}^{1}\left(\Omega, \Lambda^{l-1}\right)$, where $\gamma>0$ is a constant. Here $p^{\prime}$ is the conjugate function of $p$. Throughout this paper we suppose (unless declare specially)

$$
p \in p^{\log }(\Omega), \quad 1<p_{*}=\operatorname{essinf}_{\Omega} p(x) \leq p(x) \leq \operatorname{essup}_{\Omega} p(x)=p^{*}<\infty .
$$

\section{Preliminaries}

Let $e_{1}, e_{2}, \ldots, e_{n}$ be the standard orthogonal basis of $\mathbb{R}^{n}$. The space of all $l$-forms in $\mathbb{R}^{n}$ is denoted by $\Lambda^{l}\left(\mathbb{R}^{n}\right)$. The dual basis to $e_{1}, e_{2}, \ldots, e_{n}$ is denoted by $e^{1}, e^{2}, \ldots, e^{n}$ and referred to as the standard basis for 1-form $\Lambda^{1}\left(\mathbb{R}^{n}\right)$. The Grassman algebra $\Lambda\left(\mathbb{R}^{n}\right)=\oplus \Lambda^{l}\left(\mathbb{R}^{n}\right)$ is a graded algebra with respect to the exterior products. The standard ordered basis for $\Lambda\left(\mathbb{R}^{n}\right)$ consists of the forms

$$
1, e^{1}, e^{2}, \ldots, e^{n}, e^{1} \wedge e^{2}, \ldots, e^{n-1} \wedge e^{n}, \ldots, e^{1} \wedge e^{2} \ldots \wedge e^{n}
$$

For $\alpha(x)=\sum \alpha_{I}(x) e^{I} \in \Lambda^{l}\left(\mathbb{R}^{n}\right)$ and $\beta(x)=\sum \beta_{I}(x) e^{I} \in \Lambda^{l}\left(\mathbb{R}^{n}\right)$, the inner product is obtained by $\langle\alpha, \beta\rangle=\sum \alpha_{I}(x) \beta_{I}(x)$ with summation over all $l$-tuples $I=\left(i_{1}, \ldots i_{l}\right)$ and all integers $l=0,1, \ldots, n$. The Hodge star operator (see [14]) $\star: \Lambda\left(\mathbb{R}^{n}\right) \rightarrow \Lambda\left(\mathbb{R}^{n}\right)$ is defined by the formulas

$$
\star 1=e^{1} \wedge e^{2} \cdots \wedge e^{n}, \quad \alpha \wedge \star \beta=\beta \wedge \star \alpha=\langle\alpha, \beta\rangle e^{1} \wedge e^{2} \cdots \wedge e^{n} .
$$


Hence, the norm of $\alpha$ is given by the formula $|\alpha|^{2}=\langle\alpha, \alpha\rangle=\star(\alpha \wedge \star \alpha)=\sum \alpha_{I}(x) \alpha_{I}(x) \in$ $\Lambda^{0}\left(\mathbb{R}^{n}\right)=\mathbb{R}$. Notice, the Hodge star operator is an isometric isomorphism operator on $\Lambda\left(\mathbb{R}^{n}\right)$. Moreover,

$$
\star: \Lambda^{l}\left(\mathbb{R}^{n}\right) \longrightarrow \Lambda^{n-l}\left(\mathbb{R}^{n}\right), \quad \star \star=(-\mathrm{I})^{l(n-l)}: \Lambda^{l}\left(\mathbb{R}^{n}\right) \longrightarrow \Lambda^{l}\left(\mathbb{R}^{n}\right),
$$

where I is the identity map.

Let $\Omega \subset \mathbb{R}^{n}$ be a bounded domain. The coordinate functions $x_{1}, x_{2}, \ldots, x_{n}$ in $\Omega$ are considered to be differential forms of degree 0 . The 1 -forms $d x_{1}, d x_{2}, \ldots, d x_{n}$ are constant functions from $\Omega$ into $\Lambda^{l}\left(\mathbb{R}^{n}\right)$. The value of $d x_{i}$ is simply $e^{i}, i=1,2, \ldots, n$. Therefore, every $l$-form $u: \Omega \rightarrow \Lambda^{l}\left(\mathbb{R}^{n}\right)$ may be written uniquely as

$$
u(x)=\sum_{I} u_{I}(x) d x_{I}=\sum_{1 \leq i_{1}<\cdots<i_{l} \leq n} u_{i_{1}, \ldots i_{l}}(x) d x_{i_{1}} \wedge \cdots \wedge d x_{i_{l}}
$$

where the coefficients $u_{i_{1}, \ldots i_{l}}(x)$ are distributions from $\Phi^{\prime}(\Omega)$, dual to the space of smooth functions with compact support on $\Omega$.

We use $\Phi^{\prime}\left(\Omega, \Lambda^{l}\right)$ to denote the space of all differential $l$-forms. For each form $u(x) \in$ $\Phi^{\prime}\left(\Omega, \Lambda^{l}\right)$, the exterior differential $d: \Phi^{\prime}\left(\Omega, \Lambda^{l}\right) \rightarrow \Phi^{\prime}\left(\Omega, \Lambda^{l+1}\right)$ is expressed by

$$
d u(x)=\sum_{k=1}^{n} \sum_{1 \leq i_{1}<\cdots<i_{l} \leq n} \frac{\partial u_{i_{1}, \ldots i_{l}}(x)}{\partial x_{k}} d x_{k} \wedge d x_{i_{1}} \wedge \cdots \wedge d x_{i_{l}}
$$

For $u \in \boldsymbol{\Phi}^{\prime}\left(\Omega, \Lambda^{l}\right)$, the vector-valued differential form

$$
\nabla u=\left(\frac{\partial u}{\partial x_{1}}, \frac{\partial u}{\partial x_{2}}, \ldots, \frac{\partial u}{\partial x_{n}}\right)
$$

consists of differential forms $\partial u / \partial x_{i} \in \Phi^{\prime}\left(\Omega, \Lambda^{l}\right)$, where the partial differentiation is applied to the coefficients of $u$.

The formal adjoint operator, called the Hodge codifferential, is given by

$$
d^{\star}=(-1)^{n l-1} \star d \star: \Phi^{\prime}\left(\Omega, \Lambda^{l+1}\right) \longrightarrow \Phi^{\prime}\left(\Omega, \Lambda^{l}\right) .
$$

By $C^{\infty}\left(\Omega, \Lambda^{l}\right)$ denote the space of infinitely differentiable $l$-forms on $\Omega$ and by $C_{0}^{\infty}\left(\Omega, \Lambda^{l}\right)$ denote the subspace of $C^{\infty}\left(\Omega, \Lambda^{l}\right)$ with compact support on $\Omega$.

Let $p(\Omega)$ be the set of all Lebesgue measurable functions $p: \Omega \rightarrow(1, \infty)$. For $p \in D(\Omega)$, we put $p_{*}=\operatorname{essinf}_{\Omega} p(x)$ and $p^{*}=\operatorname{esssup}_{\Omega} p(x)$. Given $p \in D(\Omega)$ we define the conjugate function $p^{\prime} \in D(\Omega)$ by

$$
p^{\prime}(x)=\frac{p(x)}{p(x)-1}, \quad \forall x \in \Omega
$$


Definition 2.1 (see [15]). A Lebesgue measurable function $p: \Omega \rightarrow \mathbb{R}$ is called globally logHölder continuous in $\Omega$ if there exist $p_{\infty} \in \mathbb{R}$ and a constant $C>0$ such that

$$
|p(x)-p(y)| \leq \frac{C}{\log (e+1 /|x-y|)}, \quad\left|p(x)-p_{\infty}\right| \leq \frac{C}{\log (e+|x|)}
$$

hold for all $x, y \in \Omega . P^{\log }(\Omega)$ is defined by

$$
p^{\log }(\Omega)=\left\{p \in P(\Omega): \frac{1}{p} \text { is globally log-Hölder continuous }\right\} .
$$

For a differential $l$-form $u(x)$ on $\Omega, l=0,1, \ldots, n$, define the functional $\rho_{p(x)}$ by

$$
\rho_{p(x), \Lambda^{l}}(u)=\int_{\Omega}|u(x)|^{p(x)} d x
$$

The space $L^{p(x)}\left(\Omega, \Lambda^{l}\right)=\left\{u \in \Lambda^{l}(\Omega): \exists \mathcal{\lambda}>0, \rho_{p(x), \Lambda^{l}}(\mathcal{U} u)<\infty\right\}$ is a reflexive Banach space endowed with the norm

$$
\|u\|_{L^{p(x)}\left(\Omega, \Lambda^{l}\right)}=\inf \left\{\lambda>0: \rho_{p(x), \Lambda^{l}}\left(\frac{u}{\lambda}\right) \leq 1\right\}
$$

The space $W^{1, p(x)}\left(\Omega, \Lambda^{l}\right)=\left\{u \in \Lambda^{l}(\Omega): u \in L^{p(x)}\left(\Omega, \Lambda^{l}\right)\right.$ and $\left.d u \in L^{p(x)}\left(\Omega, \Lambda^{l+1}\right)\right\}$ is a reflexive Banach space endowed with the norm

$$
\|u\|_{W^{1, p(x)}\left(\Omega, \Lambda^{l}\right)}=\|u\|_{L^{p(x)}\left(\Omega, \Lambda^{l}\right)}+\|d u\|_{L^{p(x)}\left(\Omega, \Lambda^{l+1}\right)}
$$

Note that $L^{p(m)}\left(\Omega, \Lambda^{0}\right)$ and $W^{1, p(m)}\left(\Omega, \Lambda^{0}\right)$ are spaces of functions on $\Omega$. In this paper, we denote them by $L^{p(m)}(\Omega)$ and $W^{1, p(m)}(\Omega)$.

Iwaniec and Lutoborski proved the following results in [2].

Let $\Omega \subset \mathbb{R}^{n}$ be a bounded and convex domain. If $u(x) \in \Lambda^{l}\left(\mathbb{R}^{n}\right)$ is defined for some $x \in \Omega$, then the value of $u(x)$ at the vectors $\xi_{1}, \ldots, \xi_{l} \in \mathbb{R}^{n}$ is denoted by $u(x)\left(\xi_{1}, \ldots, \xi_{l}\right)$. Then to each $y \in \Omega$, there corresponds a linear operator $K_{y}: L_{\mathrm{loc}}^{1}\left(\Omega, \wedge^{l}\right) \rightarrow L_{\mathrm{loc}}^{1}\left(\Omega, \wedge^{l-1}\right)$ defined by

$$
K_{y} u(x)\left(\xi_{1}, \xi_{2}, \ldots, \xi_{l-1}\right)=\int_{0}^{1} t^{l-1} u(t x+y-t y)\left(x-y, \xi_{1}, \xi_{2}, \ldots, \xi_{l-1}\right) d t
$$

The homotopy operator $T: L_{\text {loc }}^{1}\left(\Omega, \wedge^{l}\right) \rightarrow L_{\text {loc }}^{1}\left(\Omega, \wedge^{l-1}\right)$ is defined by averaging $K_{y}$ over all points $y \in \Omega$

$$
T u(x)=\int_{\Omega} \varphi(y) K_{y} u(x) d y,
$$


where $\varphi \in C_{0}^{\infty}(\Omega)$ is normalized so that $\int_{\Omega} \varphi(y) d y=1$. Then we have a pointwise estimate

$$
|T u(x)| \leq 2^{n} \mu(\Omega) \int_{\Omega} \frac{|u(y)|}{|x-y|^{n-1}} d y, \quad \forall x \in \Omega,
$$

where

$$
\mu(\Omega)=(\operatorname{diam} \Omega)^{n+1} \inf \left\{\frac{\|\nabla \varphi\|_{L^{\infty}(\Omega)}}{\|\varphi\|_{L^{1}(\Omega)}}: \varphi \in C_{0}^{\infty}(\Omega)\right\}
$$

further infimum is attained at $\varphi(x)=\operatorname{diam}(x, \partial \Omega)$, and the decomposition

$$
u=d T u+T d u
$$

holds for $u \in L_{\text {loc }}^{1}\left(\Omega, \Lambda^{l}\right)$.

Definition 2.2. For $u \in L_{\text {loc }}^{1}\left(\Omega, \Lambda^{l}\right)$, define the $l$-form $u_{\Omega} \in \Phi^{\prime}\left(\Omega, \Lambda^{l}\right)$ by

$$
u_{\Omega}=\left\{\begin{array}{c}
\frac{1}{\operatorname{meas}(\Omega)} \int_{\Omega} u(x) d x, \quad \text { for } l=0, \\
d T u, \quad \text { for } l=1,2, \cdots, n,
\end{array}\right.
$$

and the Maximal operator is defined by

$$
(M u)(x)=\sup _{r>0} \frac{1}{\operatorname{meas}\left(B_{r}(x)\right)} \int_{B_{r}(x)}|u(y)| d y,
$$

where $B_{r}(x)=\left\{y \in \mathbb{R}^{n}:|y-x|<r\right\}$.

Lemma 2.3 (see [15]). Let $p(x)$ satisfies (1.3). Then the inequality

$$
\|(M u)(x)\|_{L^{p(x)}\left(\mathbb{R}^{n}\right)} \leq C(n, p)\|u(x)\|_{L^{p(x)}\left(\mathbb{R}^{n}\right)}
$$

holds for every $u \in L^{p(x)}\left(\mathbb{R}^{n}\right)$.

Lemma 2.4 (see [15]). Let $\Omega \subset \mathbb{R}^{n}$ be a bounded convex domain, $x \in \Omega$ and $u \in L_{\text {loc }}^{1}\left(\mathbb{R}^{n}\right)$. Then

$$
\int_{\Omega} \frac{|u(y)|}{|x-y|^{n-1}} d y \leq C(n)(\operatorname{diam} \Omega)(M u)(x)
$$


Lemma 2.5 (see [15]). Let $\Psi$ be a Calderón-Zygmund operator with Calderón-Zygmund kernel $\mathrm{K}$ on $\mathbb{R}^{n} \times \mathbb{R}^{n}$. Then $\Psi$ is bounded on $L^{p(x)}\left(\mathbb{R}^{n}\right)$. Further there exists a constant $C=C(n, p)$ such that

$$
\|\Psi u(x)\|_{L^{p(x)}\left(\mathbb{R}^{n}\right)} \leq C(n, p)\|u(x)\|_{L^{p(x)}\left(\mathbb{R}^{n}\right)}
$$

holds for every $u \in L^{p(x)}\left(\mathbb{R}^{n}\right)$.

Lemma 2.6. If $u \in L^{p(x)}\left(\Omega, \Lambda^{l}\right)$, then

$$
\|T u\|_{L^{p(x)}\left(\Omega, \Lambda^{l-1}\right)} \leq C(n, p) \mu(\Omega)(\operatorname{diam} \Omega)\|u\|_{L^{p(x)}\left(\Omega, \Lambda^{l}\right)} .
$$

Moreover, if $u \in W^{1, p(x)}\left(\Omega, \Lambda^{l}\right)$, then

$$
\left\|u_{\Omega}\right\|_{L^{p(x)}\left(\Omega, \Lambda^{l}\right)} \leq C(p)\|u\|_{L^{p(x)}\left(\Omega, \Lambda^{l}\right)}+C(n, p) \mu(\Omega)(\operatorname{diam} \Omega)\|d u\|_{L^{p(x)}\left(\Omega, \Lambda^{l+1}\right)} .
$$

Proof. First define $u(x)=0$ if $x \in \mathbb{R}^{n} \backslash \Omega$. From pointwise estimate (2.16) and Lemma 2.4,

$$
|T u(x)| \leq C(n) \mu(\Omega)(\operatorname{diam} \Omega) M(|u|)(x), \quad \forall x \in \Omega .
$$

In view of Lemma 2.3, we have

$$
\||T u|\|_{L^{p(x)}(\Omega)} \leq C(n, p) \mu(\Omega)(\operatorname{diam} \Omega)\||u|\|_{L^{p(x)}(\Omega)}
$$

that is to say, (2.24) holds.

From the definition of $u_{\Omega}$ and (2.18), we have $u_{\Omega}=u-T d u$. Therefore,

$$
\left\|u_{\Omega}\right\|_{L^{p(x)}\left(\Omega, \Lambda^{l}\right)} \leq C(p)\|u\|_{L^{p(x)}\left(\Omega, \Lambda^{l}\right)}+C(n, p)\|T d u\|_{L^{p(x)}\left(\Omega, \Lambda^{l}\right)} .
$$

Now in (2.24) replace $u$ with $d u$, we obtain (2.25).

Lemma 2.7. Let $p(x)$ satisfies (1.3).

(1) $C_{0}^{\infty}\left(\Omega, \Lambda^{l}\right)$ is dense in $L^{p(x)}\left(\Omega, \Lambda^{l}\right)$,

(2) $L^{p(x)}\left(\Omega, \Lambda^{l}\right)$ is separable.

Proof. (1) For any $u(x)=\sum_{I} u_{I}(x) d x_{I} \in L^{p(x)}\left(\Omega, \Lambda^{l}\right)$, since $C_{0}^{\infty}(\Omega)$ is dense in $L^{p(x)}(\Omega)$ and $u_{I}(x) \in L^{p(x)}(\Omega)$ for all $I$, we can find a sequence $\left\{u_{I k}\right\}_{k=1}^{\infty} \subset C_{0}^{\infty}(\Omega)$ which converges to $u_{I}(x)$ 
in $L^{p(x)}(\Omega)$ for each $I$. Now let $u_{k}(x)=\sum_{I} u_{I k} d x_{I}$, then the sequence $\left\{u_{k}(x)\right\} \subset C_{0}^{\infty}\left(\Omega, \Lambda^{l}\right)$ converges to $u(x)$ in $L^{p(x)}\left(\Omega, \Lambda^{l}\right)$, since

$$
\begin{aligned}
\int_{\Omega}\left|u(x)-u_{k}(x)\right|^{p(x)} d x & =\int_{\Omega}\left(\left(\sum_{I}\left|u_{I}(x)-u_{I k}(x)\right|^{2}\right)^{1 / 2}\right)^{p(x)} d x \\
& \leq \int_{\Omega}\left(\sum_{I}\left|u_{I}(x)-u_{I k}(x)\right|\right)^{p(x)} d x \\
& \leq 2^{p^{*}} \sum_{I} \int_{\Omega}\left|u_{I}(x)-u_{I k}(x)\right|^{p(x)} d x .
\end{aligned}
$$

That is to say, $C_{0}^{\infty}\left(\Omega, \Lambda^{l}\right)$ is dense in $L^{p(x)}\left(\Omega, \Lambda^{l}\right)$.

(2) Let $u(x)=\sum_{I} u_{I}(x) d x_{I} \in L^{p(x)}\left(\Omega, \Lambda^{l}\right)$. Since $L^{p(x)}(\Omega)$ is separable, there exists a countable dense subset $K$ of $L^{p(x)}(\Omega)$. Then for any $u_{I}(x)$ above we can extract a sequence $\left\{u_{I k}(x)\right\}$ in $K$ which converges to $u_{I}(x)$ in $L^{p(x)}(\Omega)$. Similar to (1), the sequence $\left\{u_{k}: u_{k}(x)=\right.$ $\left.\sum_{I} u_{I k}(x) d x_{I}\right\}$ converges to $u(x)$ in $L^{p(x)}\left(\Omega, \Lambda^{l}\right)$. That is to say, $L^{p(x)}\left(\Omega, \Lambda^{l}\right)$ is separable.

Let $\mathfrak{K}^{1, p(x)}\left(\Omega, \Lambda^{l}\right)=\left\{u(x)=\vartheta(x)-\vartheta_{\Omega}(x): \vartheta \in W^{1, p(x)}\left(\Omega, \Lambda^{l}\right)\right\}$. Note that $u \in$ $\mathfrak{K}^{1, p(x)}\left(\Omega, \Lambda^{l}\right)$ if and only if $u_{\Omega}=0$.

Lemma 2.8. Let $p(x)$ satisfies (1.3). Then $\mathfrak{K}^{1, p(x)}\left(\Omega, \Lambda^{l}\right)$ is a closed subspace of $W^{1, p(x)}\left(\Omega, \Lambda^{l}\right)$. In particular, it is a reflexive Banach space.

Proof. Set a sequence $\left\{u_{k}(x)\right\} \subset \mathfrak{K}^{1, p(x)}\left(\Omega, \Lambda^{l}\right)$ convergent to $u(x)$ in $W^{1, p(x)}\left(\Omega, \Lambda^{l}\right)$, then $\left(u_{k}\right)_{\Omega}=0$. By Lemma 2.6, the operator $T$ is continuous on $\mathfrak{K}^{1, p(x)}\left(\Omega, \Lambda^{l}\right)$. Therefore, $u_{\Omega}=$ 0 , we have $u(x) \in \mathfrak{K}^{1, p(x)}\left(\Omega, \Lambda^{l}\right)$. That is to say, $\mathfrak{K}^{1, p(x)}\left(\Omega, \Lambda^{l}\right)$ is a closed subspace of $W^{1, p(x)}\left(\Omega, \Lambda^{l}\right)$.

In [2], Iwaniec and Lutoborski obtained

$$
\frac{\partial}{\partial x_{i}}(T u)=A_{i} u+S_{i} u
$$

where

$$
\begin{gathered}
\left|A_{i} u(x)\right| \leq \frac{2^{n} \mu(\Omega)}{\operatorname{diam}(\Omega)} \int_{\Omega} \frac{|u(z)|}{|x-z|^{n-1}} d z, \\
S_{i} u(x)(\xi)=\int_{\Omega} u(z)\left(K_{i}(z, x-z), \xi\right) d z,
\end{gathered}
$$


where $\xi=\left(\xi_{1}, \xi_{2}, \ldots, \xi_{l-1}\right)$ and

$$
\begin{aligned}
K_{i}(z, x-z) & =\frac{e_{i}}{|x-z|^{n}} \int_{0}^{\infty} s^{n-1} \varphi\left(z-s \frac{x-z}{|x-z|}\right) d s \\
& -\frac{x-z}{|x-z|^{n+1}} \int_{0}^{\infty} s^{n} \varphi_{i}\left(z-s \frac{x-z}{|x-z|}\right) d s .
\end{aligned}
$$

Further for each $z \in \Omega$ and $h \in \mathbb{R}^{n}-\{0\}, K_{i}(z, h)$ satisfies the following properties:

(i) $K_{i}(z, h) \leq \mu(\Omega)|h|^{-n}$,

(ii) $K_{i}(z, s h)=s^{-n} K_{i}(z, h), s>0$,

(iii) $\int_{|h|=1} K_{i}(z, h)=0$ for all $z \in \Omega$.

Let $K_{i}(z, h)=\left(K_{i 1}, K_{i 2}, \ldots, K_{i n}\right)$. Then $K_{i \alpha}$ satisfies the conditions of CalderónZygmund kernel on $\mathbb{R}^{n} \times \mathbb{R}^{n}$ for each $\alpha=1,2, \ldots, n$.

Lemma 2.9. Let $u \in L^{p(x)}\left(\Omega, \Lambda^{l}\right)$. Then

$$
\||\nabla T u|\|_{L^{p(x)}(\Omega)} \leq C(n, p, \Omega)\|u\|_{L^{p(x)}\left(\Omega, \Lambda^{l}\right)} .
$$

Proof. By Lemmas 2.3 and 2.4, and (2.31),

$$
\left\|A_{i} u\right\|_{L^{p(x)}\left(\Omega, \Lambda^{l}\right)} \leq C(n, p) \mu(\Omega)\|u\|_{L^{p(x)}\left(\Omega, \Lambda^{l}\right)} .
$$

Let

$$
S_{i} u(x)=\sum_{1 \leq j_{1}<j_{2}<\cdots<j_{l-1} \leq n} \omega_{j_{1}, j_{2}, \ldots, j_{l-1}} d x_{j_{1}} \wedge d x_{j_{2}} \wedge \cdots \wedge d x_{j_{l-1}}
$$

we can write $u(x)$ as

$$
u(x)=\sum_{1 \leq \alpha \leq n, \alpha \neq j_{1}, j_{2}, \ldots, j_{l-1} 1 \leq j_{1}<j_{2}<\cdots<j_{l-1} \leq n} u_{\alpha, j_{1}, j_{2}, \ldots, j_{l-1}} d x_{\alpha} \wedge d x_{j_{1}} \wedge \cdots \wedge d x_{j_{l-1}} .
$$

Hence,

$$
\omega_{j_{1}, j_{2}, \ldots, j_{l-1}}(x)=S_{i} u(x)\left(e_{j_{1}}, e_{j_{2}}, \ldots, e_{j_{l-1}}\right) .
$$

Taking $\xi=\left(e_{j_{1}}, e_{j_{2}}, \ldots, e_{j_{l-1}}\right)$ in (2.32), we obtain

$$
\omega_{j_{1}, j_{2}, \ldots, j_{l-1}}(x)=\int_{\Omega} \sum_{1 \leq \alpha \leq n, \alpha \neq j_{1}, \ldots, j_{l-1}} K_{i \alpha}(z, x-z) u_{\alpha, j_{1}, j_{2}, \ldots, j_{l-1}}(z) d z
$$


Now define $u(x)=0 \quad$ if $\quad x \in \mathbb{R}^{n} \backslash \Omega$. Since $K_{i \alpha}$ satisfies the conditions of CalderónZygmund kernel on $\mathbb{R}^{n} \times \mathbb{R}^{n}$ for each $\alpha$, in view of Lemma 2.5,

$$
\left\|\omega_{j_{1}, j_{2}, \ldots, j_{l-1}}\right\|_{L^{p(x)}(\Omega)} \leq C(n, p) \sum_{1 \leq \alpha \leq n, \alpha \neq j_{1}, \ldots, j_{l-1}}\left\|u_{\alpha, j_{1}, j_{2}, \ldots, j_{l-1}}\right\|_{L^{p(x)}(\Omega)}
$$

So that

$$
\left\|S_{i} u\right\|_{L^{p(x)}\left(\Omega, \Lambda^{l}\right)} \leq C(n, p)\|u\|_{L^{p(x)}\left(\Omega, \Lambda^{l}\right)} .
$$

By (2.30), (2.35), and (2.41), we have

$$
\||\nabla T u|\|_{L^{p(x)}(\Omega)} \leq C(n, p, \Omega)\|u\|_{L^{p(x)}\left(\Omega, \Lambda^{l}\right)}
$$

Now define another norm

$$
\||\omega|\|_{\mathfrak{K}^{1, p(x)}\left(\Omega, \Lambda^{l}\right)}=\|\omega\|_{L^{p(x)}\left(\Omega, \Lambda^{l}\right)}+\||\nabla \omega|\|_{L^{p(x)}(\Omega)} .
$$

Remark 2.10. Replacing $u$ with $d u$ in (2.34), we get by the definition of $u_{\Omega}$

$$
\begin{aligned}
\left\|\left|\nabla\left(u-u_{\Omega}\right)\right|\right\|_{L^{p(x)}(\Omega)} & =\||\nabla T d u|\|_{L^{p(x)}(\Omega)} \\
& \leq C(n, p) \mu(\Omega)\|d u\|_{L^{p(x)}\left(\Omega, \Lambda^{l}\right)} \\
& =C(n, p) \mu(\Omega)\left\|d\left(u-u_{\Omega}\right)\right\|_{L^{p(x)}\left(\Omega, \Lambda^{l}\right)} .
\end{aligned}
$$

Therefore $\||\cdot|\|_{\mathfrak{K}^{1, p(x)}\left(\Omega, \Lambda^{l}\right)}$ is equivalent to $\|\cdot\|_{\mathfrak{K}^{1, p(x)}\left(\Omega, \Lambda^{l}\right)}$.

In this paper we also need the following two lemmas.

Lemma 2.11 (see[15]). Let $p(x)$ satisfies (1.3). Then the embedding $W_{0}^{1, p(x)}(\Omega) \hookrightarrow L^{p(x)}(\Omega)$ is compact.

Lemma 2.12 (see[15]). Suppose that $p \in L^{\infty}(\Omega)$. Let $\left\{u_{k}\right\}_{k=1}^{\infty}$ be bounded in $L^{p(x)}(\Omega)$. If $u_{k} \rightarrow u$ a.e. on $\Omega$, then $u_{k} \rightarrow u$ weakly in $L^{p(x)}(\Omega)$.

Remark 2.13. Let $\mathfrak{K}_{0}^{1, p(x)}\left(\Omega, \Lambda^{l}\right)$ be the completion of $C_{0}^{\infty}\left(\Omega, \Lambda^{l}\right)$ in $\mathfrak{K}^{1, p(x)}\left(\Omega, \Lambda^{l}\right)$. Then from Remark 2.10 and Lemma 2.11, the embedding $\mathfrak{K}_{0}^{1, p(x)}\left(\Omega, \Lambda^{l}\right) \hookrightarrow L^{p(x)}\left(\Omega, \Lambda^{l}\right)$ is compact.

Remark 2.14. Suppose $p(x)$ satisfies (1.3), Lemma 2.12 also holds on space $L^{p(x)}\left(\Omega, \wedge^{l}\right)$. 


\section{Weak Solutions of Dirichlet Problems for the $A$-Harmonic Equations with Variable Growth}

Theorem 3.1. Under conditions (H1)-(H5), the Dirichlet problem (1.1) has at least one weak solution in $\mathfrak{K}_{0}^{1, p(x)}\left(\Omega, \Lambda^{l}\right)$, that is to say, there exists at least one $u=\vartheta-\vartheta_{\Omega} \in \mathfrak{K}_{0}^{1, p(x)}\left(\Omega, \Lambda^{l}\right)$ satisfying

$$
\int_{\Omega}\langle A(x, d u(x)), d \varphi(x)\rangle+\langle B(x, u(x)), \varphi(x)\rangle d x=0
$$

for all $\varphi \in W_{0}^{1, p(x)}\left(\Omega, \Lambda^{l-1}\right)$. Here, $\vartheta \in W^{1, p(x)}\left(\Omega, \Lambda^{l-1}\right)$ and $p(x)$ satisfies (1.3).

Let $V=W_{0}^{1, p(x)}\left(\Omega, \Lambda^{l-1}\right)$ and $\mathfrak{K}_{0}=\mathfrak{K}_{0}^{1, p(x)}\left(\Omega, \Lambda^{l}\right)$. For $u \in V$, define $\mathfrak{A}: V \rightarrow V^{*}$ in the following way: for each $\varphi \in V$

$$
(\mathfrak{A} u, \varphi)=\int_{\Omega}\langle A(x, d u(x)), d \varphi(x)\rangle+\langle B(x, u(x)), \varphi(x)\rangle d x
$$

Now we need only to show that there exists $u \in \mathfrak{K}_{0}$ such that $(\mathfrak{A} u, \varphi)=0$ for all $\varphi=$ $\sum \varphi_{I}(x) d x_{I} \in V$.

Lemma 3.2. $\mathfrak{A}$ is strong-weakly continuous on $V$.

Proof. Let $\left\{u_{k}: u_{k}(x)=\sum_{I} u_{k I}(x) d x_{I}\right\} \subset V$ be a sequence strongly convergent to an element $u(x)=\sum u_{I}(x) d x_{I} \in V$ in $V$. Let $d u_{k}(x)=\sum_{J} \omega_{k J}(x) d x_{J}$ and $d u(x)=\sum_{J} \omega_{J}(x) d x_{J}$. Then

$\left(h_{1}\right)\left\|u_{k}\right\|_{V} \leq C$ for some constant $C$,

$\left(h_{2}\right)\left\{\omega_{k J}(x)\right\}$ is a sequence strongly convergent to $\omega_{J}(x)$ in $L^{p(x)}(\Omega)$ for each $J$.

In view of (H2) and $\left(h_{1}\right)$, we know that $A\left(x, d u_{k}\right)=\sum A_{k J}(x) d x_{J}$ and $B\left(x, u_{k}\right)=$ $\sum B_{k I}(x) d x_{I}$ are uniformly bounded in $L^{p^{\prime}(x)}\left(\Omega, \Lambda^{l}\right)$ and $L^{p^{\prime}(x)}\left(\Omega, \Lambda^{l-1}\right)$, respectively. Hence, $A_{k J}(x)$ and $B_{k I}(x)$ are uniformly bounded in $L^{p^{\prime}(x)}(\Omega)$. On the other hand, by $\left(h_{2}\right)$, there exists a subsequence of $\left\{\omega_{k J}(x)\right\}$ (still denoted by $\left.\left\{\omega_{k J}(x)\right\}\right)$ such that

$$
\lim _{k \rightarrow \infty} \omega_{k J}(x)=\omega_{J}(x), \text { a.e. } x \in \Omega \text {, for each } J
$$

Then there exists a subsequence of $\left\{u_{k}(x)\right\}$ (still denoted by $\left.\left\{u_{k}(x)\right\}\right)$ such that

$$
\lim _{k \rightarrow \infty} u_{k}(x)=u(x), \quad \lim _{k \rightarrow \infty} d u_{k}(x)=d u(x), \quad \text { a.e. } x \in \Omega
$$

In view of (H1), we obtain

$$
\begin{gathered}
\lim _{k \rightarrow \infty} A\left(x, d u_{k}\right)=A(x, d u), \quad \text { a.e. } x \in \Omega, \\
\lim _{k \rightarrow \infty} B\left(x, u_{k}\right)=B(x, u), \quad \text { a.e. } x \in \Omega .
\end{gathered}
$$


Let $d \varphi(x)=\sum \psi_{J}(x) d x_{J}, A(x, d u)=\sum A_{J}(x) d x_{J}$, and $B(x, u)=\sum B_{I}(x) d x_{I}$, then $\psi_{J}(x) \in$ $L^{p(x)}(\Omega)$, in the meantime

$$
\begin{array}{ll}
\lim _{k \rightarrow \infty} A_{k J}(x)=A_{J}(x), & \text { a.e. } x \in \Omega, \\
\lim _{k \rightarrow \infty} B_{k I}(x)=B_{I}(x), & \text { a.e. } x \in \Omega,
\end{array}
$$

for each $J$ and $I$.

Now by Lemma 2.12, we can show that $\int_{\Omega} A_{k J}(x) \psi_{J}(x) d x \rightarrow \int_{\Omega} A_{J}(x) \psi_{J}(x) d x$ and $\int_{\Omega} B_{k I}(x) \varphi_{I}(x) d x \rightarrow \int_{\Omega} B_{I}(x) \varphi_{I}(x) d x$ as $k \rightarrow \infty$. Therefore,

$$
\begin{aligned}
\left(\mathfrak{A} u_{k}, \varphi\right) & =\int_{\Omega}\left\langle A\left(x, d u_{k}\right), d \varphi\right\rangle+\left\langle B\left(x, u_{k}\right), \varphi\right\rangle d x \\
& \longrightarrow \int_{\Omega}\langle A(x, d u), d \varphi\rangle+\langle B(x, u), \varphi\rangle d x \\
& =(\mathfrak{A} u, \varphi),
\end{aligned}
$$

that is to say, $\mathfrak{A}$ is strong-weakly continuous on $V$.

Lemma 3.3. $\mathfrak{A}$ is coercive on $\mathfrak{K}_{0}$, that is,

$$
\lim _{\|u\|_{\mathfrak{K}} \rightarrow \infty} \frac{(\mathfrak{A} u, u)}{\|u\|_{\mathfrak{K}}}=+\infty, \quad \forall u \in \mathfrak{K}_{0}
$$

Proof. By (H3) and (H4),

$$
\begin{aligned}
(\mathfrak{A} u, u) & =\int_{\Omega}\langle A(x, d u), d u\rangle+\langle B(x, u), u\rangle d x \\
& \geq \int_{\Omega}\left(a|d u|^{p(x)}-|h(x)|+\bar{a}|u|^{p(x)}-|\bar{h}(x)|\right) d x \\
& \geq \int_{\Omega} a|d u|^{p(x)} d x-C(h, \bar{h}) .
\end{aligned}
$$

By $d v_{\Omega}=0$ and Lemma 2.6, we have

$$
\|u\|_{L^{p(x)}\left(\Omega, \Lambda^{l-1}\right)}=\|T d \vartheta\|_{L^{p(x)}\left(\Omega, \Lambda^{l-1}\right)} \leq 2^{n} C(n, p) \mu(\Omega)(\operatorname{diam} \Omega)\|d u\|_{L^{p(x)}\left(\Omega, \Lambda^{l}\right)},
$$

for all $u=\vartheta-\vartheta_{\Omega} \in \mathfrak{K}_{0}$. Then $\|d u\|_{L^{p(x)}\left(\Omega, \Lambda^{l}\right)} \rightarrow \infty$, as $\|u\|_{\mathfrak{K}} \rightarrow \infty$. Taking

$$
\delta=\frac{1}{2}\|d u\|_{L^{p(x)}\left(\Omega, \Lambda^{l}\right)}>1,
$$


we have

$$
\begin{aligned}
\frac{\int_{\Omega}|d u|^{p(x)} d x}{\|d u\|_{L^{p(x)}\left(\Omega, \Lambda^{l}\right)}} & =\int_{\Omega}\left(\frac{|d u|}{\|d u\|_{L^{p(x)}\left(\Omega, \Lambda^{l}\right)}-\delta}\right)^{p(x)} \frac{\left(\|d u\|_{L^{p(x)}\left(\Omega, \Lambda^{l}\right)}-\delta\right)^{p(x)}}{\|d u\|_{L^{p(x)}\left(\Omega, \Lambda^{l}\right)}} d x \\
& \geq \frac{\left(\|d u\|_{L^{p(x)}\left(\Omega, \Lambda^{l}\right)}-\delta\right)^{p_{*}}}{\|d u\|_{L^{p(x)}\left(\Omega, \Lambda^{l}\right)}}=\left(\frac{1}{2}\right)^{p_{*}}\|d u\|_{L^{p(x)}\left(\Omega, \Lambda^{l}\right)^{p_{*}-1}} .
\end{aligned}
$$

Therefore,

$$
\frac{\int_{\Omega}|d u|^{p(x)} d x}{\|d u\|_{L^{p(x)}\left(\Omega, \Lambda^{l}\right)}} \longrightarrow \infty \quad \text { as }\|d u\|_{L^{p(x)}\left(\Omega, \Lambda^{l}\right)} \longrightarrow \infty
$$

Then it is immediate to obtain that

$$
\frac{(\mathfrak{A} u, u)}{\|u\|_{\mathfrak{K}}} \longrightarrow \infty \quad \text { as }\|u\|_{\mathfrak{K}} \longrightarrow \infty
$$

That is to say, $\mathfrak{A}$ is coercive on $\mathfrak{K}_{0}$.

Lemma 3.4 (see[16]). Suppose $g=A(x)$ is a mapping from $\mathbb{R}^{m}$ into itself such that

$$
\lim _{|x| \rightarrow \infty} \frac{A(x) \cdot x}{|x|}=\infty
$$

Then the range of $A$ is the whole of $\mathbb{R}^{m}$.

Lemma 3.5. There exists a sequence $\left\{u_{k}\right\} \subset \mathfrak{K}_{0}$ and $u_{0} \in \mathfrak{K}_{0}$, such that

$$
\left(\mathfrak{A} u_{k}, u_{k}-u_{0}\right) \longrightarrow 0 \text { as } k \longrightarrow \infty
$$

Proof. By Lemmas 2.7 and 2.8, we can choose a Schauder basis $\left\{\omega_{s}\right\}$ of $\mathfrak{K}_{0}$ such that the union of subspace finitely generated from $\omega_{s}$ is dense in $\mathfrak{K}_{0}$. Let $\mathfrak{K}_{0}^{k}$ be the subspace of $\mathfrak{K}_{0}$ generated by $\omega_{1}, \omega_{2}, \ldots, \omega_{k}$. Since $\mathfrak{K}_{0}^{k}$ is topologically isomorphic to $\mathbb{R}^{k}$. By By Lemmas 3.3, and 3.4, there exists $u_{k} \in \mathfrak{K}_{0}^{k}$ such that

$$
\left(\mathfrak{A} u_{k}, \omega\right)=0 \quad \forall \omega \in \mathfrak{K}_{0}^{k}
$$

By Lemma 3.3 again, we know that $\left\|u_{k}\right\|_{\mathfrak{K}} \leq C$, where $C$ is independent of $k$. Since $\mathfrak{K}_{0}$ is reflexive, by Remark 2.14 and (H1), we can extract a subsequence of $\left\{u_{k}\right\}$ (still denoted by $\left.\left\{u_{k}\right\}\right)$ such that

$$
u_{k} \rightarrow u_{0} \quad \text { weakly in } \mathfrak{K}_{0}, \quad \mathfrak{A} u_{k} \rightarrow \xi \quad \text { weakly }{ }^{*} \text { in } \mathfrak{K}_{0}^{*}, \quad(\xi, \omega)=0,
$$


where $\omega$ is in a dense subset of $\mathfrak{K}_{0}$. For fixed $\xi$, by the continuity of $(\xi, \cdot)$, we get $(\xi, \omega)=0$ for all $\omega \in \mathfrak{K}_{0}$. For $\left(\mathfrak{A} u_{k}, u_{k}-u_{0}\right)$, we have

$$
\left(\mathfrak{A} u_{k}, u_{k}-u_{0}\right)=\left(\mathfrak{A} u_{k}, u_{k}\right)-\left(\mathfrak{A} u_{k}, u_{0}\right)=-\left(\mathfrak{A} u_{k}, u_{0}\right) \longrightarrow 0 \text { as } k \longrightarrow \infty
$$

This completes the proof of Lemma 3.5.

$$
\begin{aligned}
& \text { Set } v_{k}=u_{k}-u_{0}=\sum v_{k I} d x_{I} \text {. Then } \\
& \qquad v_{k} \rightarrow 0 \quad \text { weakly in } \mathfrak{K}_{0} \quad \text { as } k \longrightarrow \infty
\end{aligned}
$$

Consider $\left(\mathfrak{A} u_{k}, u_{k}-u_{0}\right)$ once more, then

$$
\left(\mathfrak{A} u_{k}, u_{k}-u_{0}\right)=\int_{\Omega}\left\langle A\left(x, d u_{0}+d v_{k}\right), d v_{k}\right\rangle+\left\langle B\left(x, u_{0}+v_{k}\right), v_{k}\right\rangle d x \rightarrow 0
$$

as $k \rightarrow \infty$. By Remark 2.13, we get

$$
v_{k} \longrightarrow 0 \text { strongly in } L^{p(x)}\left(\Omega, \Lambda^{l-1}\right)
$$

In view of (3.23) and (H2), it is immediate that

$$
\int_{\Omega}\left\langle B\left(x, u_{0}+v_{k}\right), v_{k}\right\rangle d x \longrightarrow 0 \text { as } k \longrightarrow \infty
$$

that is to say,

$$
\int_{\Omega}\left\langle A\left(x, d u_{0}+d v_{k}\right), d v_{k}\right\rangle d x \longrightarrow 0 \quad \text { as } k \longrightarrow \infty
$$

Now if we can prove that there exists a subsequence of $\left\{v_{k}\right\}$ which is strongly convergent in $\mathfrak{K}_{0}$, then from the strong-weakly continuity of $\mathfrak{A}$, we get $\mathfrak{A} u_{k} \rightarrow \mathfrak{A} u_{0}=\xi$ weakly in $\mathfrak{K}_{0}$ as $k \rightarrow \infty$ and $u_{0}$ will be a weak solution of (1.1). We need the following lemmas.

Definition 3.6. Let $\Omega$ be an open subset of $\mathbb{R}^{n}$ provided with the Lebesgue measure. The mapping $f: \Omega \times \mathbb{R}^{N} \rightarrow \mathbb{R}$ is said Carathéodory function if for almost all $x \in \Omega, f(x, \cdot)$ is continuous on $\mathbb{R}^{N}$, for all $\xi \in \mathbb{R}^{N}$ is measurable on $\Omega$.

Lemma 3.7 (see[17]). A mapping $f: \Omega \times \mathbb{R}^{N} \rightarrow \mathbb{R}$ is a Carathéodory function if and only if for all compact sets $K \subset \Omega$ and all $\varepsilon>0$, there exists a compact subset $K_{\varepsilon} \subset K$ such that meas $\left(K-K_{\varepsilon}\right)<\varepsilon$ for with the restriction of $f$ to $K_{\varepsilon} \times \mathbb{R}^{N}$ is continuous. 
Lemma 3.8 (see[15]). Let $\left\{f_{k}\right\}$ be a sequence of bounded function in $L^{1}\left(\mathbb{R}^{n}\right)$. For each $\varepsilon>0$ there exists $\left(A_{\varepsilon}, \delta, N\right)$ (where $A_{\varepsilon}$ is measurable and meas $\left(A_{\varepsilon}\right)<\varepsilon, \delta>0, N$ is an infinite subset of natural numbers set $\mathbb{N}$ ) such that for each $k \in N$,

$$
\int_{B}\left|f_{k}(x)\right| d x<\varepsilon
$$

where $B$ and $A_{\varepsilon}$ are disjoint and meas $(B)<\delta$.

Definition 3.9. For $u \in C_{0}^{1}\left(\mathbb{R}^{n}\right)$, define

$$
\left(M^{*} u\right)(x)=(M u)(x)+\sum_{\alpha=1}^{n}\left(M \frac{\partial u}{\partial x_{\alpha}}\right)(x)
$$

Lemma 3.10 (see[18]). If $u \in C_{0}^{\infty}\left(\mathbb{R}^{n}\right)$, then $M^{*} u \in C^{0}\left(\mathbb{R}^{n}\right)$ and for all $x \in \mathbb{R}^{n}$,

$$
|u(x)|+\sum_{\alpha=1}^{n}\left|\frac{\partial u}{\partial x_{\alpha}}(x)\right| \leq\left(M^{*} u\right)(x)
$$

Furthermore, if $p>1$, then

$$
\left\|M^{*} u\right\|_{L^{p}\left(\mathbb{R}^{n}\right)} \leq C(n, p)\|u\|_{W^{1, p}\left(\mathbb{R}^{n}\right)}
$$

and if $p=1$, then

$$
\operatorname{meas}\left(\left\{x \in \mathbb{R}^{n}:\left(M^{*} u\right)(x)>\lambda\right\}\right) \leq \frac{C(n)}{\lambda}\|u\|_{W^{1,1}\left(\mathbb{R}^{n}\right)},
$$

for all $\lambda>0$.

Lemma 3.11 (see[19]). Let $u \in C_{0}^{\infty}\left(\mathbb{R}^{n}\right)$ and $\lambda>0$. Set

$$
H^{\lambda}=\left\{x \in \mathbb{R}^{n}:\left(M^{*} u\right)(x)<\lambda\right\}
$$

Then for all $x, y \in H^{\curlywedge}$, we have

$$
|u(y)-u(x)| \leq C(n) \lambda|y-x|
$$

Lemma 3.12 (see[16]). Let $X$ be a metric space, $E$ be a subspace of $X$, and $k$ be a positive number. Then any $k$-Lipchitz mapping from $E$ into $\mathbb{R}$ can be extended to a $k$-Lipchitz mapping from $X$ into $\mathbb{R}$.

Proof of Theorem 3.1. We need only to show that there exists subsequence of $\left\{v_{k}\right\}$ which is strongly convergent in $\mathfrak{K}_{0}$. 
For each measurable set $S \subset \Omega$, define

$$
F(v, S)=\int_{S}\left\langle A\left(x, d u_{0}+d v\right), d v\right\rangle d x
$$

where $v \in \mathfrak{K}_{0}$. Similar to the proof of Lemma 3.2, $F(\cdot, S)$ is strongly continuous on $\mathfrak{K}_{0}$. Since $C_{0}^{\infty}\left(\Omega, \Lambda^{l-1}\right)$ is dense in $\mathfrak{K}_{0}$, there exists $h_{k} \subset C_{0}^{\infty}\left(\Omega, \Lambda^{l-1}\right)$ such that

$$
\left\|h_{k}-v_{k}\right\|_{\mathfrak{K}}<\frac{1}{k}, \quad\left|F\left(h_{k}, \Omega\right)-F\left(v_{k}, \Omega\right)\right|<\frac{1}{k} .
$$

So we can suppose that $\left\{v_{k}\right\} \subset C_{0}^{\infty}\left(\Omega, \Lambda^{l-1}\right)$ is bounded in $\mathfrak{K}_{0}$.

Next define

$$
v_{k}(x)=0 \quad \text { when } x \in \mathbb{R}^{n} \backslash \Omega \text {. }
$$

In this way, we extend the domain of $v_{k}$ to $\mathbb{R}^{n}$ and supp $v_{k} \subset \Omega$.

Let $\beta: \mathbb{R}^{+} \rightarrow \mathbb{R}^{+}$be a continuous increasing function satisfying $\beta(0)=0$ and for each measurable set $D \subset \Omega$,

$$
\int_{D}\left(|G(x)|^{p^{\prime}(x)}+|h(x)|+\left(C_{1}+1\right)\left|d u_{0}\right|^{p(x)}\right) d x \leq \beta(\operatorname{meas}(D))
$$

where $C_{1}$ is the constant in ( $\left.\mathrm{H} 2\right)$.

Let $\left\{\varepsilon_{j}\right\}$ be a positive decreasing sequence with $\varepsilon_{j} \rightarrow 0$ as $j \rightarrow \infty$. For $\varepsilon_{1}$, by Lemma 3.8, we get a subsequence $\left\{k_{1}\right\}$ of $\{k\}$, a set $A_{\varepsilon_{1}} \subset \Omega$ satisfying meas $\left(A_{\varepsilon_{1}}\right)<\varepsilon_{1}$, and a real number $\delta_{1}>0$ such that

$$
\int_{B}\left(M^{*} v_{k_{1} I}\right)^{p(x)} d x<\varepsilon_{1}
$$

for each $k_{1}, I$ and $B \subset \Omega \backslash A_{\varepsilon_{1}}$ satisfying meas $(B)<\delta_{1}$. By Lemma 3.10, we can choose $\lambda>1$ so large that for all $I$ and $k_{1}$,

$$
\operatorname{meas}\left(\left\{x \in \mathbb{R}^{n}:\left(M^{*} v_{k_{1} I}\right)(x) \geq \lambda\right\}\right) \leq \min \left\{\varepsilon_{1}, \delta_{1}\right\}
$$

For each $I$ and $k_{1}$, define

$$
H_{k_{1} I}^{\lambda}=\left\{x \in \mathbb{R}^{n}:\left(M^{*} v_{k_{1} I}\right)(x)<\lambda\right\}, \quad H_{k_{1}}^{\lambda}=\bigcap_{I} H_{k_{1} I}^{\lambda} .
$$

In view of Lemma 3.11, we have

$$
\frac{\left|v_{k_{1} I}(y)-v_{k_{1} I}(x)\right|}{|y-x|} \leq C(n) \lambda \quad \forall x, y \in H_{k_{1}}^{\lambda} \text { and } I .
$$


Form Lemma 3.12, there exists a Lipschitz function $g_{k_{1} I}$ which extends $v_{k_{1} I}$ outside $H_{k_{1}}^{\lambda}$ and Lipschitz constant of $g_{k_{1} I}$ is no more than $C(n) \lambda$. As $H_{k_{1}}^{\lambda}$ is an open set, we have $g_{k_{1} I}=v_{k_{1} I}$ and $\nabla g_{k_{1} I}(x)=\nabla v_{k_{1} I}(x)$ for all $x \in H_{k_{1}}^{\lambda}$, and $\left\|\nabla g_{k_{1} I}\right\|_{L^{\infty}\left(\mathbb{R}^{n}\right)} \leq C(n) \lambda$. We can further suppose that

$$
\left\|g_{k_{1} I}\right\|_{L^{\infty}\left(\mathbb{R}^{n}\right)} \leq\left\|v_{k_{1} I}\right\|_{L^{\infty}\left(H_{k_{1}}^{\lambda}\right)} \leq \lambda, \quad\left\|g_{k_{1} I}\right\|_{W^{1, \infty}(\Omega)} \leq C(n) \lambda .
$$

By the uniformly boundedness of $\left\{\left\|g_{k_{1} I}\right\|_{W^{1, \infty}(\Omega)}\right\}$, there exists a subsequence of $\left\{g_{k_{1} I}\right\}$ (still denoted by $\left.\left\{g_{k_{1} I}\right\}\right)$ such that

$$
g_{k_{1} I} \rightarrow \omega_{I} \text { weakly }^{*} \text { in } W^{1, \infty}(\Omega) \text { as } k_{1} \longrightarrow \infty \forall I .
$$

Set $\omega=\sum_{I} \omega_{I} d x_{I}$ and $g_{k_{1}}=\sum_{I} g_{k_{1} I} d x_{I}$. We have

$$
F\left(v_{k_{1}}, \Omega\right)=F\left(g_{k_{1}}, \Omega \backslash A_{\varepsilon_{1}}\right)-F\left(g_{k_{1}},\left(\Omega \backslash A_{\varepsilon_{1}}\right) \backslash H_{k_{1}}^{\lambda}\right)+F\left(v_{k_{1}}, A_{\varepsilon_{1}} \cup\left(\Omega \backslash H_{k_{1}}^{\lambda}\right)\right) .
$$

Next we estimate $F\left(v_{k_{1}}, \Omega\right)$ in four steps.

(1) The estimate of $F\left(g_{k_{1}},\left(\Omega \backslash A_{\varepsilon_{1}}\right) \backslash H_{k_{1}}^{\lambda}\right)$ and $F\left(v_{k_{1}}, A_{\varepsilon_{1}} \cup\left(\Omega \backslash H_{k_{1}}^{\lambda}\right)\right)$. Since

$$
\operatorname{meas}\left(\left(\Omega \backslash A_{\varepsilon_{1}}\right) \backslash H_{k_{1}}^{\lambda}\right) \leq \sum_{I} \operatorname{meas}\left(\left(\Omega \backslash A_{\varepsilon_{1}}\right) \backslash H_{k_{1} I}^{\lambda}\right) \leq \mathrm{C}_{n}^{l-1} \min \left\{\varepsilon_{1}, \delta_{1}\right\},
$$

where $C_{n}^{l-1}=n(n-1) \cdots(n-l+2) /(l-1)(l-2) \cdots 1$, from (H2), (H3), and the choose of $A_{\varepsilon_{1}}$, we have

$$
\begin{aligned}
& \left|F\left(g_{k_{1}},\left(\Omega \backslash A_{\varepsilon_{1}}\right) \backslash H_{k_{1}}^{\lambda}\right)\right| \\
& \quad \leq \int_{\left(\Omega \backslash A_{\varepsilon_{1}}\right) \backslash H_{k_{1}}^{\lambda}}\left(C_{1}\left|d u_{0}+d g_{k_{1}}\right|^{p(x)-1}\left|d g_{k_{1}}\right|+|G(x)|\left|d g_{k_{1}}\right|\right) d x \\
& \quad \leq \int_{\left(\Omega \backslash A_{\varepsilon_{1}}\right) \backslash H_{k_{1}}^{\lambda}}\left(C_{1} 2^{p^{*}-1}\left(\left|d u_{0}\right|^{p(x)}+\left|d g_{k_{1}}\right|^{p(x)}\right)+C_{1}\left|d g_{k_{1}}\right|^{p(x)}+|G(x)|^{p^{\prime}(x)}+\left|d g_{k_{1}}\right|^{p(x)}\right) d x \\
& \quad \leq 2^{p^{*}-1} \beta\left(\operatorname{meas}\left(\left(\Omega \backslash A_{\varepsilon_{1}}\right) \backslash H_{k_{1}}^{\lambda}\right)\right)+2^{p^{*}}\left(C_{1}+1\right) \int_{\left(\Omega \backslash A_{\varepsilon_{1}}\right) \backslash H_{k_{1}}^{\lambda}}\left|d g_{k_{1}}\right|^{p(x)} d x \\
& \quad \leq 2^{p^{*}-1} \beta\left(\operatorname{meas}\left(\left(\Omega \backslash A_{\varepsilon_{1}}\right) \backslash H_{k_{1}}^{\lambda}\right)\right)+2^{p^{*}}\left(C_{1}+1\right) \int_{\left(\Omega \backslash A_{\varepsilon_{1}}\right) \backslash H_{k_{1}}^{\lambda}}\left(\sum_{I}\left|\nabla g_{k_{1} I}\right|\right)^{p(x)} d x
\end{aligned}
$$


Abstract and Applied Analysis

$$
\begin{aligned}
& \leq 2^{p^{*}-1} \beta\left(C_{n}^{l-1} \varepsilon_{1}\right)+2^{p^{*}} C\left(C_{1}, n, l\right) \int_{\left(\Omega \backslash A_{\varepsilon_{1}}\right) \backslash H_{k_{1}}^{\lambda}} \lambda^{p(x)} d x \\
& \leq 2^{p^{*}-1} \beta\left(C_{n}^{l-1} \varepsilon_{1}\right)+2^{p^{*}} C\left(C_{1}, n, l\right) \sum_{I} \int_{\left(\Omega \backslash A_{\varepsilon_{1}}\right) \backslash H_{k_{1} I}^{\lambda}}\left(M^{*} v_{k_{1} I}\right)^{p(x)} d x \\
\leq & 2^{p^{*}-1} \beta\left(C_{n}^{l-1} \varepsilon_{1}\right)+2^{p^{*}} C\left(C_{1}, n, l\right) \varepsilon_{1} \leq O\left(\varepsilon_{1}\right), \\
F\left(v_{k_{1}}, A_{\varepsilon_{1}} \cup\left(\Omega \backslash H_{k_{1}}^{\lambda}\right)\right) & \\
= & \int_{A_{\varepsilon_{1}} \cup\left(\Omega \backslash H_{k_{1}}^{\lambda}\right)}\left\langle A\left(x, d u_{0}+d v_{k_{1}}\right), d u_{0}+d v_{k_{1}}\right\rangle-\left\langle A\left(x, d u_{0}+d v_{k_{1}}\right), d u_{0}\right\rangle d x \\
\geq & \int_{A_{\varepsilon_{1}} \cup\left(\Omega \backslash H_{k_{1}}^{\lambda}\right)}\left(a\left|d u_{0}+d v_{k_{1}}\right|^{p(x)}-h(x)\right)-\left(C_{1}\left|d u_{0}+d v_{k_{1}}\right|^{p(x)-1}\left|d u_{0}\right|+|G(x)|\left|d u_{0}\right|\right) d x \\
\geq & \int_{A_{\varepsilon_{1}} \cup\left(\Omega \backslash H_{k_{1}}^{\lambda}\right)}\left(\left(a 2^{-\left(p^{*}-1\right)}-C_{1} \mu 2^{p^{*}-1}\right)\left|d v_{k_{1}}\right|^{p(x)}-|h(x)|-|G(x)|^{p^{\prime}(x)}\right) \\
& -\left(-a 2^{-\left(p^{*}-1\right)}+C_{1} \mu 2^{p^{*}-1}+C_{1} C(\mu)+1\right)\left|d u_{0}\right|^{p(x)} d x \\
\geq & \left(a 2^{-\left(p^{*}-1\right)}-C_{1} \mu 2^{p^{*}-1}\right) \int_{A_{\varepsilon_{1}} \cup\left(\Omega \backslash H_{k_{1}}^{\lambda}\right)}\left|d v_{k_{1}}\right|^{p(x)} d x-C\left(a, p, C_{1}, \mu\right) \beta\left(\operatorname{meas}\left(A_{\varepsilon_{1}} \cup\left(\Omega \backslash H_{k_{1}}^{\lambda}\right)\right)\right) \\
\geq & a 2^{-p^{*}} \int_{A_{\varepsilon_{1}} \cup\left(\Omega \backslash H_{k_{1}}^{\lambda}\right)}\left|d v_{k_{1}}\right|^{p(x)} d x-O\left(\varepsilon_{1}\right),
\end{aligned}
$$

where $\mu>0$ is small enough.

From (3.43)-(3.46), we get

$$
F\left(v_{k_{1}}, \Omega\right) \geq F\left(g_{k_{1}}, \Omega \backslash A_{\varepsilon_{1}}\right)+a 2^{-p^{*}} \int_{A_{\varepsilon_{1}} \cup\left(\Omega \backslash H_{k_{1}}^{\lambda}\right)}\left|d v_{k_{1}}\right|^{p(x)} d x-O\left(\varepsilon_{1}\right) .
$$

(2) The estimate of $F\left(g_{k_{1}}, \Omega \backslash A_{\varepsilon_{1}}\right)$. Set $f_{k_{1} I}=g_{k_{1} I}-\omega_{I}$, where $\omega_{I}$ is defined by (3.42). Then

$$
\begin{gathered}
f_{k_{1} I} \rightarrow 0 \text { weakly }{ }^{*} \text { in } W^{1, \infty}(\Omega) \quad \text { as } k_{1} \longrightarrow \infty \quad \forall I, \\
\left\|f_{k_{1} I}\right\|_{L^{\infty}(\Omega)} \leq 2 \lambda, \quad\left\|d f_{k_{1} I}\right\|_{L^{\infty}\left(\Omega, \Lambda^{l}\right)} \leq 2 C(n) \lambda .
\end{gathered}
$$


Let $G=\bigcup_{I} G_{I}$ with $G_{I}=\left\{x \in \Omega: \omega_{I}(x) \neq 0\right\}$. According to Acerbi and Fusco [19], we have meas $(G) \leq\left(C_{n}^{l-1}+1\right) \varepsilon_{1}$ where $C_{n}^{l-1}=n(n-1) \cdots(n-l+2) /(l-1)(l-2) \cdots 1$, and set $f_{k_{1}}=\sum_{I} f_{k_{1} I} d x_{I}$, then

$$
\begin{aligned}
F\left(g_{k_{1}}, \Omega \backslash A_{\varepsilon_{1}}\right)= & F\left(f_{k_{1}},\left(\Omega \backslash A_{\varepsilon_{1}}\right) \backslash G\right) \\
& +F\left(v_{k_{1}},\left(\Omega \backslash A_{\varepsilon_{1}}\right) \cap H_{k_{1}}^{\lambda} \cap G\right) \\
& +F\left(g_{k_{1}},\left(\Omega \backslash A_{\varepsilon_{1}}\right) \cap\left(G \backslash H_{k_{1}}^{\lambda}\right)\right) .
\end{aligned}
$$

Define

$$
\begin{aligned}
& \Omega_{1}^{\varepsilon_{1}, k_{1}}=A_{\varepsilon_{1}} \cup\left(\Omega \backslash H_{k_{1}}^{\lambda}\right), \quad \Omega_{2}^{\varepsilon_{1}}=\left(\Omega \backslash A_{\varepsilon_{1}}\right) \backslash G, \\
& \Omega_{3}^{\varepsilon_{1}, k_{1}}=\left(\Omega \backslash A_{\varepsilon_{1}}\right) \cap H_{k_{1}}^{\lambda} \cap G, \quad \Omega_{4}^{\varepsilon_{1}, k_{1}}=\left(\Omega \backslash A_{\varepsilon_{1}}\right) \cap\left(G \backslash H_{k_{1}}^{\lambda}\right) .
\end{aligned}
$$

Similar to the proof of (3.46), we get

$$
F\left(v_{k_{1}}, \Omega_{3}^{\varepsilon_{1}, k_{1}}\right) \geq a 2^{-p^{*}} \int_{\Omega_{3}^{\varepsilon_{1}, k_{1}}}\left|d v_{k_{1}}\right|^{p(x)} d x-O\left(\varepsilon_{1}\right)
$$

Since on $\Omega_{4}^{\varepsilon_{1}, k_{1}}$ we have

$$
\int_{\Omega_{4}^{\varepsilon_{1}, k_{1}}}\left|d g_{k_{1}}\right|^{p(x)} d x \leq C(n, p)\left(C_{n}^{l-1}+1\right) \varepsilon_{1}
$$

then similar to the proof of (3.45), we get

$$
\left|F\left(g_{k_{1}}, \Omega_{4}^{\varepsilon_{1}, k_{1}}\right)\right| \leq O\left(\varepsilon_{1}\right)
$$

By (3.49)-(3.53), we have

$$
F\left(g_{k_{1}}, \Omega \backslash A_{\varepsilon_{1}}\right) \geq F\left(f_{k_{1}}, \Omega_{2}^{\varepsilon_{1}}\right)+a 2^{-p^{*}} \int_{\Omega_{3}^{\varepsilon_{1}, k_{1}}}\left|d v_{k_{1}}\right|^{p(x)} d x-O\left(\varepsilon_{1}\right) .
$$

Thus, we have

$$
F\left(v_{k_{1}}, \Omega\right) \geq F\left(f_{k_{1}}, \Omega_{2}^{\varepsilon_{1}}\right)+a 2^{-p^{*}} \int_{\Omega_{5}^{\varepsilon_{1}}, k_{1}}\left|d v_{k_{1}}\right|^{p(x)} d x-O\left(\varepsilon_{1}\right),
$$

where $\Omega_{5}^{\varepsilon_{1}, k_{1}}=\Omega_{1}^{\varepsilon_{1}, k_{1}} \cup \Omega_{3}^{\varepsilon_{1}, k_{1}}$. 
Choose an open set $\Omega^{\prime} \subset \Omega$ which contains $\Omega_{2}^{\varepsilon_{1}}$ such that

$$
\left|F\left(f_{k_{1}}, \Omega^{\prime}\right)-F\left(f_{k_{1}}, \Omega_{2}^{\varepsilon_{1}}\right)\right|<\varepsilon_{1} .
$$

From (3.55), we get

$$
F\left(v_{k_{1}}, \Omega\right) \geq F\left(f_{k_{1}}, \Omega^{\prime}\right)+a 2^{-p^{*}} \int_{\Omega_{5}^{\varepsilon_{1}, k_{1}}}\left|d v_{k_{1}}\right|^{p(x)} d x-O\left(\varepsilon_{1}\right) .
$$

Approximate $\Omega^{\prime}$ by hypercubes with edges parallel to coordinate axes, that is, construct

$$
\begin{aligned}
H_{j} & \subset \Omega^{\prime}, \\
\operatorname{meas}\left(\Omega^{\prime} \backslash H_{j}\right) & \longrightarrow 0 \text { as } j \longrightarrow \infty, \\
H_{j} & =\bigcup_{s=1}^{h_{j}} D_{j, s}, \\
\operatorname{meas}\left(D_{j, s}\right) & =1 / 2^{n j}, \quad 1 \leq s \leq h_{j} .
\end{aligned}
$$

Let $j>0$ be large enough such that for all $k_{1}>0$, we have

$$
\begin{gathered}
\left|F\left(f_{k_{1}}, \Omega^{\prime}\right)-F\left(f_{k_{1}}, H_{j}\right)\right|<\varepsilon_{1}, \quad \int_{\Omega^{\prime} \backslash H_{j}}\left|d f_{k_{1}}\right|^{p(x)} d x<\varepsilon_{1}, \\
\operatorname{meas}\left(\Omega^{\prime} \backslash H_{j}\right)<\min \left\{\varepsilon_{1}, \delta_{1}\right\} .
\end{gathered}
$$

Thus,

$$
F\left(v_{k_{1}}, \Omega\right) \geq F\left(f_{k_{1}}, H_{j}\right)+a 2^{-p^{*}} \int_{\Omega_{5}^{\varepsilon_{1}, k_{1}}}\left|d v_{k_{1}}\right|^{p(x)} d x-O\left(\varepsilon_{1}\right)-2 \varepsilon_{1}
$$

(3) The estimate of $F\left(f_{k_{1}}, H_{j}\right)$. Let $\alpha>0$ be large enough such that for $E=\left\{x \in \Omega^{\prime}\right.$ : $\eta(x) \leq \alpha\}$. Then

$$
\operatorname{meas}\left(\Omega^{\prime} \backslash E\right)<\frac{\varepsilon_{1}}{N}, \quad \int_{\Omega^{\prime} \backslash E} \eta(x) d x<\varepsilon_{1},
$$

where $\left\|d f_{k_{1}}\right\|_{L^{\infty}\left(\Omega, \Lambda^{l}\right)} \leq 2 C_{n}^{l-1} C(n) \lambda=N$ and $\eta(x)=|G(x)|^{p^{\prime}(x)}+2^{p^{*}-1}\left(C_{1}+1\right)\left|d u_{0}\right|^{p(x)}$.

For $x \in \Omega, \xi \in \Lambda^{l}(\Omega)$, define

$$
\psi(x, \xi)=\left\langle A\left(x, d u_{0}(x)+\xi\right), \xi\right\rangle .
$$


By Lemma 3.7 and (H1), there exists a compact subset $K \subset H_{j}$ such that $\psi(x, \xi)$ is continuous on $K \times \Lambda^{l}(\Omega)$ and meas $\left(H_{j} \backslash K\right)<\varepsilon_{1} /(\alpha+N)$. Hence, $\psi(x, \xi)$ is uniformly continuous on bounded subsets of $K \times \Lambda^{l}(\Omega)$.

Divide each $D_{j, s}$ into $2^{n m}$ hypercubes $Q_{t, j, s}^{m}$ with edge length $2^{-j m}, 1 \leq t \leq 2^{n m}$. For all $j, s, m, t$, take $x_{t, j, s}^{m} \in Q_{t, j, s}^{m} \cap K \cap E$ (if this set is empty, take $x_{t, j, s}^{m} \in Q_{t, j, s}^{m}$ ) such that

$$
\eta\left(x_{t, j, s}^{m}\right) \operatorname{meas}\left(Q_{t, j, s}^{m}\right) \leq \int_{Q_{t, j, s}^{m}} \eta(x) d x
$$

Then

$$
\begin{aligned}
F( & \left.f_{k_{1}}, H_{j}\right) \\
= & F\left(f_{k_{1}}, H_{j} \cap K \cap E\right)+F\left(f_{k_{1}}, H_{j} \backslash E\right)+F\left(f_{k_{1}},\left(H_{j} \cap E\right) \backslash K\right) \\
\geq & F\left(f_{k_{1}}, H_{j} \cap K \cap E\right)-\int_{H_{j} \backslash E} \eta(x) d x-\int_{\left(H_{j} \cap E\right) \backslash K} \eta(x) d x \\
& -2^{p^{*}}\left(C_{1}+1\right)\left(\int_{H_{j} \backslash E}\left|d f_{k_{1}}\right|^{p(x)} d x+\int_{\left(H_{j} \cap E\right) \backslash K}\left|d f_{k_{1}}\right|^{p(x)} d x\right) \\
= & F\left(f_{k_{1}}, H_{j} \cap K \cap E\right)-O\left(\varepsilon_{1}\right) \\
= & b_{k_{1}}^{m, j}+c_{k_{1}}^{m, j}+d_{k_{1}}^{m, j}-O\left(\varepsilon_{1}\right),
\end{aligned}
$$

where

$$
\begin{aligned}
b_{k_{1}}^{m, j} & =\sum_{t, s} \int_{Q_{t, j, s}^{m} \cap K \cap E}\left(\psi\left(x, d f_{k_{1}}(x)\right)-\psi\left(x_{t, j, s^{\prime}}^{m} d f_{k_{1}}(x)\right)\right) d x, \\
c_{k_{1}}^{m, j} & =\sum_{t, s} \int_{Q_{t, j, s}^{m}} \psi\left(x_{t, j, s}^{m} d f_{k_{1}}(x)\right) d x \\
d_{k_{1}}^{m, j} & =-\sum_{t, s} \int_{Q_{t, j, s}^{m} \backslash(K \cap E)} \psi\left(x_{t, j, s}^{m} d f_{k_{1}}(x)\right) d x .
\end{aligned}
$$

By (3.25), we have

$$
\lim _{k_{1} \rightarrow \infty} F\left(v_{k_{1}}, \Omega\right)=0
$$

Note that if $Q_{t, j, s}^{m} \cap K \cap E$ is an empty set, then

$$
\int_{Q_{t, j, s}^{m} \cap K \cap E}\left[\psi\left(x, d f_{k_{1}}(x)\right)-\psi\left(x_{t, j, s}^{m}, d f_{k_{1}}(x)\right)\right] d x=0 .
$$


Now we only consider $Q_{t, j, s}^{m}$ which satisfies $Q_{t, j, s}^{m} \cap K \cap E \neq \phi$. Since $d u_{0}(x)$ is uniformly continuous on $H_{j}$, then by the uniform continuity of $\psi$ on bounded subsets of $K \times \Lambda^{l}(\Omega)$, we obtain that for $x \in Q_{t, j, s}^{m}$, there exists a constant $L>0$ such that

$$
\begin{aligned}
\mid \psi(x, & \left.d f_{k_{1}}(x)\right)-\psi\left(x_{t, j, s^{\prime}}^{m} d f_{k_{1}}(x)\right) \mid \\
& =\left|\left\langle A\left(x, d u_{0}(x)+d f_{k_{1}}(x)\right)-A\left(x_{t, j, s}^{m} d u_{0}\left(x_{t, j, s}^{m}\right)+d f_{k_{1}}(x)\right), d f_{k_{1}}(x)\right\rangle\right| \\
& <\frac{1}{\operatorname{meas}\left(H_{j}\right)} \varepsilon_{1}
\end{aligned}
$$

holds for all $m>L$ and each $k_{1}$. Therefore, $\left|b_{k_{1}}^{m, j}\right|<\varepsilon_{1}$ for all $k_{1}$.

$$
\begin{aligned}
\left|d_{k_{1}}^{m, j}\right| & \leq \sum_{t, s} \int_{Q_{t, j, s}^{m} \backslash(K \cap E)}\left|\psi\left(x_{t, j, s^{\prime}}^{m} d f_{k_{1}}(x)\right)\right| d x \\
& =\sum_{t, s} \int_{Q_{t, j, s}^{m} \backslash(K \cap E)}\left\langle A\left(x_{t, j, s^{\prime}}^{m} d u_{0}\left(x_{t, j, s}^{m}\right)+d f_{k_{1}}(x)\right), d f_{k_{1}}(x)\right\rangle d x \\
& \leq \sum_{t, s} \int_{Q_{t, j, s}^{m} \backslash(K \cap E)} C_{1}\left|d u_{0}\left(x_{t, j, s}^{m}\right)+d f_{k_{1}}(x)\right|^{p(x)-1}\left|d f_{k}(x)\right|+\left|G\left(x_{t, j, s}^{m}\right)\right| d f_{k_{1}}(x) \mid d x \\
& \leq \sum_{t, s} \int_{Q_{t, j, s}^{m} \backslash(K \cap E)}\left(\eta\left(x_{t, j, s}^{m}\right)+2^{p^{*}}\left(C_{1}+1\right) N\right) d x \\
& \leq \int_{\left(H_{j} \cap E\right) \backslash K}\left(\eta\left(x_{t, j, s}^{m}\right)+2^{p^{*}}\left(C_{1}+1\right) N\right) d x+C\left(C_{1}, p\right) \sum_{t, s} \int_{Q_{t, j, s}^{m} \backslash E}\left(\eta\left(x_{t, j, s}^{m}\right)+N\right) d x \\
& \leq C\left(\alpha, N, C_{1}, p\right) \operatorname{meas}\left(\left(H_{j} \cap E\right) \backslash K\right)+C\left(C_{1}, p\right) \int_{H_{j} \backslash E}[\eta(x)+N] d x \\
& \leq C\left(\alpha, N, C_{1}, p\right) \varepsilon_{1} \leq O\left(\varepsilon_{1}\right) .
\end{aligned}
$$

Now we suppose that $m$ is large enough that $\left|b_{k_{1}}^{m, j}\right|<\varepsilon_{1}$ for each $k_{1}>0$ and there exists $\bar{k}_{1}>0$ such that $F\left(v_{k_{1}} \Omega\right)<\varepsilon_{1}$ for $k_{1}>\bar{k}_{1}$. Therefore, from (3.25), (3.60), and (3.64), we have

$$
\begin{aligned}
\varepsilon_{1} & \geq F\left(v_{k_{1}}, \Omega\right) \\
& \geq c_{k_{1}}^{m, j}+a 2^{-p^{*}} \int_{\Omega_{5}^{\varepsilon_{1}, k_{1}}}\left|d v_{k_{1}}\right|^{p(x)} d x-O\left(\varepsilon_{1}\right)-3 \varepsilon_{1}-C\left(C_{1}, p\right) \varepsilon_{1} \\
& =c_{k_{1}}^{m, j}+a 2^{-p^{*}} \int_{\Omega_{5}^{\varepsilon_{1}, k_{1}}}\left|d v_{k_{1}}\right|^{p(x)} d x-O\left(\varepsilon_{1}\right) .
\end{aligned}
$$


(4) The estimate of $c_{k_{1}, j}^{m}$. By $f_{k_{1} I} \rightarrow 0$ weakly ${ }^{*}$ in $W^{1, \infty}(\Omega)$ as $k_{1} \rightarrow \infty$, we obtain $\left\|f_{k_{1} I}\right\|_{L^{\infty}(\Omega)} \rightarrow 0$ ask $\rightarrow \infty$ for each $I$. Then

$$
R_{t, s, j}^{k_{1, m}}=\left\|\left|f_{k_{1}}\right|\right\|_{L^{\infty}\left(Q_{t, s, j}^{m}\right)} \longrightarrow 0 \quad \text { as } k_{1} \longrightarrow \infty \text { for fixed } m
$$

Define a hypercube $E_{t, s, j}^{k_{1, m}}$ contained in $Q_{t, s, j}^{m}$ with edge length $1 / 2^{j m}-2 R_{t, s, j}^{k_{1}, m}$ such that $\operatorname{dist}\left(\partial Q_{t, s, j}^{m}, E_{t, s, j}^{k_{1}, m}\right)=R_{t, s, j}^{k_{1}, m}$.

Next define

$$
\begin{gathered}
\varphi_{k_{1}}(x)=0, \quad x \in \partial Q_{t, s, j}^{m} \\
\varphi_{k_{1}}(x)=f_{k_{1}}(x), \quad x \in E_{t, s, j}^{m} .
\end{gathered}
$$

Since $\varphi_{k_{1} I}$ is a Lipschitz mapping on set $E_{t, s, j}^{m} \cup \partial Q_{t, s, j}^{m}$ and its Lipschitz constant is no more than $2 C(n) \lambda$, by Lemma 3.12, $\varphi_{k_{1} I}$ can be extended to the whole $Q_{t, s, j}^{m}$, where it is also a Lipschitz mapping with the same Lipchistz constant. We still denote the extension by $\varphi_{k_{1} I}$ and suppose that it is defined on the whole $H_{j}$. Then by [20]

$$
\nabla \varphi_{k_{1} I}-\nabla f_{k_{1} I} \longrightarrow 0 \quad \text { a.e. on } H_{j}
$$

Thus, there exists a $\overline{\overline{k_{1}}}>\overline{k_{1}}$ such that for all $k_{1}>\overline{\overline{k_{1}}}$, we have

$$
\begin{gathered}
\int_{H_{j}}\left|d \varphi_{k_{1}}-d f_{k_{1}}\right|^{p(x)} d x \leq \frac{\varepsilon_{1}}{2} \\
\sum_{t, s}\left|\int_{Q_{t, j, s}^{m}} \psi\left(x_{t, j, s^{\prime}}^{m} d f_{k_{1}}(x)\right)-\psi\left(x_{t, j, s^{\prime}}^{m} d \varphi_{k_{1}}(x)\right) d x\right| \leq \frac{\varepsilon_{1}}{2} .
\end{gathered}
$$

In view of (H5), we obtain that

$$
\begin{aligned}
c_{k_{1}}^{m, j} & =\sum_{t, s} \int_{Q_{t, j, s}^{m}} \psi\left(x_{t, j, s}^{m}, d f_{k_{1}}(x)\right) d x \\
& \geq \sum_{t, s} \int_{Q_{t, j, s}^{m}} \psi\left(x_{t, j, s}^{m}, d \varphi_{k_{1}}(x)\right) d x-\frac{\varepsilon_{1}}{2} \\
& =\sum_{t, s} \int_{Q_{t, j, s}^{m}}\left\langle A\left(x_{t, j, s}^{m} d u_{0}\left(x_{t, j, s}^{m}\right)+d \varphi_{k_{1}}(x)\right), d \varphi_{k_{1}}(x)\right\rangle d x-\frac{\varepsilon_{1}}{2} \\
& \geq \gamma \sum_{t, s} \int_{Q_{t, j, s}^{m}}\left|d \varphi_{k_{1}}\right|^{p(x)} d x-\frac{\varepsilon_{1}}{2} \\
& \geq \frac{\gamma}{2^{p^{*}-1}} \int_{H_{j}}\left|d f_{k_{1}}\right|^{p(x)} d x-\frac{(\gamma+1) \varepsilon_{1}}{2} .
\end{aligned}
$$


Thus in (3.70) for $k_{1}>\overline{\overline{k_{1}}}$, we obtain the estimate of $F\left(v_{k_{1}}, \Omega\right)$ from the four steps above

$$
\begin{aligned}
\varepsilon_{1} & \geq F\left(v_{k_{1}}, \Omega\right) \\
& \geq a 2^{-p^{*}} \int_{\Omega_{5}^{\varepsilon_{1}, k_{1}}}\left|d v_{k_{1}}\right|^{p(x)} d x+\frac{\gamma}{2^{p^{*}-1}} \int_{H_{j}}\left|d f_{k_{1}}\right|^{p(x)} d x-\frac{(\gamma+1) \varepsilon_{1}}{2}-O\left(\varepsilon_{1}\right) .
\end{aligned}
$$

Let $K\left(\varepsilon_{1}\right)=(\gamma+1) \varepsilon_{1} /\left(2+o\left(\varepsilon_{1}\right)\right) / \min \left\{a 2^{-p^{*}}, \gamma / 2^{p^{*}-1}\right\}$. Then

$$
\int_{\Omega_{5}^{\varepsilon_{1}, k_{1}}}\left|d v_{k_{1}}\right|^{p(x)} d x+\int_{H_{j}}\left|d f_{k_{1}}\right|^{p(x)} d x \leq K\left(\varepsilon_{1}\right), \quad \text { for } k_{1}>\overline{\overline{k_{1}}} \text {. }
$$

Form (3.59) and (3.77), we deduce that

$$
\int_{\Omega_{5}^{\varepsilon_{1}, k_{1}}}\left|d v_{k_{1}}\right|^{p(x)} d x \leq K\left(\varepsilon_{1}\right), \quad \int_{\Omega^{\prime}}\left|d f_{k_{1}}\right|^{p(x)} d x \leq K\left(\varepsilon_{1}\right)+\varepsilon_{1} .
$$

According to the definition of $\Omega_{2}^{\varepsilon_{1}}$, we have

$$
\int_{\Omega_{\varepsilon_{1}}^{2}}\left|d g_{k_{1}}\right|^{p(x)} d x \leq K\left(\varepsilon_{1}\right)+\varepsilon_{1}
$$

Since $d g_{k_{1}}(x)=d v_{k_{1}}(x)$ for each $x \in H_{k_{1}}^{\lambda}$, we get

$$
\int_{\Omega_{\varepsilon_{1}}^{2} \cap H_{k_{1}}^{\lambda}}\left|d v_{k_{1}}\right|^{p(x)} d x \leq K\left(\varepsilon_{1}\right)+\varepsilon_{1}
$$

By the definitions of $\Omega_{2}^{\varepsilon_{1}}$ and $\Omega_{5}^{\varepsilon_{1}, k_{1}}$, it is immediate that

$$
\left(\Omega_{2}^{\varepsilon_{1}} \cap H_{k_{1}}^{\lambda}\right) \cup \Omega_{5}^{\varepsilon_{1}, k_{1}}=\Omega,
$$

which implies that

$$
\int_{\Omega}\left|d v_{k_{1}}\right|^{p(x)} d x \leq 2 K\left(\varepsilon_{1}\right)+\varepsilon_{1} \leq O\left(\varepsilon_{1}\right) .
$$

For $\varepsilon_{2}>0$ and the sequence $\left\{v_{k_{1}}\right\}$, repeating the above arguments we can extract a subsequence $\left\{v_{k_{2}}\right\}$ of $\left\{v_{k_{1}}\right\}$ such that

$$
\int_{\Omega}\left|d v_{k_{2}}\right|^{p(x)} d x \leq O\left(\varepsilon_{2}\right),
$$


whenever $k_{2}>\overline{\overline{k_{2}}}$ for some $\overline{\overline{k_{2}}}$. If $\left\{v_{k_{n}}\right\}$ has been obtained, repeating the above process, we can extract a subsequence $\left\{k_{n+1}\right\}$ of $\left\{k_{n}\right\}$ such that

$$
\int_{\Omega}\left|d v_{k_{n+1}}\right|^{p(x)} d x \leq O\left(\varepsilon_{n+1}\right)
$$

whenever $k_{n+1}>\overline{\bar{k}}_{n+1}$ for some $\overline{\bar{k}}_{n+1}$. Finally, by a diagonal argument we get a subsequence $\left\{v_{k_{i}}\right\}_{i=1}^{\infty}$ which satisfies

$$
\int_{\Omega}\left|d v_{k_{i}}\right|^{p(x)} d x \longrightarrow 0 \quad \text { as } i \longrightarrow \infty
$$

Therefore,

$$
\left\|d v_{k_{i}}\right\|_{L^{p(x)}\left(\Omega, \Lambda^{l}\right)} \longrightarrow 0 \quad \text { as } i \longrightarrow \infty,
$$

and by (3.23), $\left\{v_{k_{i}}\right\}_{i=1}^{\infty}$ strongly converges to zero in $\mathfrak{K}_{0}$ as $i \rightarrow \infty$. This completes the proof of Theorem 3.1.

\section{Applications}

In this section, we explore applications of our results developed in this paper.

Let $\Omega \subset \mathbb{R}^{n}$ be a bounded and convex Lipschitz domain. Suppose that maps $A: \Omega \times$ $\Lambda^{l}(\Omega) \rightarrow \Lambda^{l}(\Omega)$ and $B: \Omega \times \Lambda^{l-1}(\Omega) \rightarrow \Lambda^{l-1}(\Omega)$, where $l=1,2, \ldots, n$.

Example 4.1. If $p(x)$ satisfies (1.3), let $l=1, A(x, \xi)=\left.\xi|\xi|\right|^{p(x)-2}$ and $B(x, \varsigma)=\varsigma \mid \varsigma^{p(x)-2}-f(x)$, where $f(x) \in L^{p^{\prime}(x)}(\Omega)$. Then $A, B$ satisfy the required conditions, and (1.1) reduce to the following $p(x)$-Laplacian equations:

$$
\begin{gathered}
-\operatorname{div}\left(|\nabla u|^{p(x)-2} \nabla u\right)+|u|^{p(x)-2} u=f(x), \quad x \in \Omega, \\
u(x)=0, \quad x \in \partial \Omega .
\end{gathered}
$$

Now by Theorem 3.1, we deduce that the $p(x)$-Laplacian equations (4.1) have at least one weak solution in $\mathfrak{K}^{1, p(x)}(\Omega)$ with $u=0$ on $\partial \Omega$.

Example 4.2. If $l=1, A(x, \xi)=\sum_{i, j} A_{i j}(x) \xi_{j} d x_{i}, B(x, \varsigma)=B(x) \varsigma-f(x)$, where $f(x) \in L^{2}(\Omega)$, and $A_{i j}(x), B(x)$ satisfy the following conditions:

$$
A_{i j}(x)=A_{j i}(x), \quad \wedge|\xi|^{2} \geq \sum_{i, j=1}^{n} A_{i j}(x) \xi_{i} \xi_{j} \geq \lambda|\xi|^{2}, \quad \lambda \leq B(x) \leq \wedge
$$


for some constants $\lambda, \wedge>0$. Then $A, B$ satisfy the required conditions, and (1.1) reduce to the following Divergence form equations:

$$
\begin{gathered}
\sum_{i, j=1}^{n} \nabla_{j}\left(A_{i j}(x) \nabla_{i} u(x)\right)+B(x) u(x)=f(x), \quad x \in \Omega, \\
u(x)=0, \quad x \in \partial \Omega,
\end{gathered}
$$

where $\nabla_{i}=\left(\partial / \partial x_{i}\right)$. Now by Theorem 3.1, we deduce that the divergence form (4.3) have at least one weak solution $u(x)$ in $\mathfrak{K}^{1,2}(\Omega)$ with $u=0$ on $\partial \Omega$. The comparison principles, the maximum principles, and the existence of weak solutions for divergence form equation (4.3) can be found in [21].

Example 4.3. If $p(x)$ satisfies (1.3), let $A(x, \xi)=\xi|\xi|^{p(x)-2}$ and $B(x, \varsigma)=\varsigma|\varsigma|^{p(x)-2}-f(x)$, where $f(x) \in L^{p^{\prime}(x)}\left(\Omega, \wedge^{l-1}\right)$. Then $A, B$ satisfy the required conditions, and (1.1) reduce to the following $p(x)$-harmonic equations for differential forms:

$$
\begin{gathered}
d^{*}\left(d u|d u|^{p(x)-2}\right)+u|u|^{p(x)-2}=f(x), \quad x \in \Omega, \\
u(x)=0, \quad x \in \partial \Omega .
\end{gathered}
$$

Now by Theorem 3.1, we deduce that (4.5) have at least one weak solution $u(x)$ in $\mathfrak{K}_{0}^{1, p(x)}\left(\Omega, \wedge^{l-1}\right)$. If $p(x)$ is a constant $q$ and $1<q<\infty$, the equation (4.5) is called nonhomogeneous $q$-harmonic equation. In [2], Iwaniec and Lutoborski studied the $L^{q}$ theory of weak solution for homogeneous $q$-harmonic equations.

\section{References}

[1] V. M. Gol'dshteĭn, V. I. Kuz'minov, and I. A. Shvedov, "Dual spaces to spaces of differential forms," Akademiya Nauk SSSR, vol. 27, no. 1, pp. 35-44, 1986.

[2] T. Iwaniec and A. Lutoborski, "Integral estimates for null Lagrangians," Archive for Rational Mechanics and Analysis, vol. 125, no. 1, pp. 25-79, 1993.

[3] F. L. Teixeira and W. C. Chew, "Differential forms, metrics, and the reflectionless absorption of electromagnetic waves," Journal of Electromagnetic Waves and Applications, vol. 13, no. 5, pp. 665-686, 1999.

[4] F. L. Teixeira, "Differential form approach to the analysis of electromagnetic cloaking and masking," Microwave and Optical Technology Letters, vol. 49, no. 8, pp. 2051-2053, 2007.

[5] P. Morando, "Liouville condition, Nambu mechanics, and differential forms," Journal of Physics A, vol. 29, no. 13, pp. L329-L331, 1996.

[6] H. C. J. Sealey, "Some conditions ensuring the vanishing of harmonic differential forms with applications to harmonic maps and Yang-Mills theory," Mathematical Proceedings of the Cambridge Philosophical Society, vol. 91, no. 3, pp. 441-452, 1982.

[7] S. Ding and C. A. Nolder, "Weighted Poincaré inequalities for solutions to A-harmonic equations," Illinois Journal of Mathematics, vol. 46, no. 1, pp. 199-205, 2002.

[8] R. P. Agarwal and S. Ding, "Advances in differential forms and the A-harmonic equation," Mathematical and Computer Modelling, vol. 37, no. 12-13, pp. 1393-1426, 2003.

[9] Y. Xing and S. Ding, "Caccioppoli inequalities with Orlicz norms for solutions of harmonic equations and applications," Nonlinearity, vol. 23, no. 5, pp. 1109-1119, 2010.

[10] Y. Xing and S. Ding, "Norms of the composition of the maximal and projection operators," Nonlinear Analysis: Theory, Methods E Applications, vol. 72, no. 12, pp. 4614-4624, 2010.

[11] S. Ding and B. Liu, "Global estimates for singular integrals of the composite operator," Illinois Journal of Mathematics, vol. 53, no. 4, pp. 1173-1185, 2009. 
[12] O. Kováčik and J. Rákosník, “On spaces $L^{p(x)}$ and $W^{k, p(x)}$, " Czechoslovak Mathematical Journal, vol. 41(116), no. 4, pp. 592-618, 1991.

[13] Y. Fu and L. Guo, "Weighted variable exponent spaces of differential forms and their applications".

[14] G. de Rham, Differentiable Manifolds, vol. 266 of Grundlehren der Mathematischen Wissenschaften, Springer, Berlin, Germany, 1984.

[15] L. Diening, P. Harjulehto, P. Hästö, and M. Ružička, Lebesgue and Sobolev Spaces with Variable Exponents, vol. 2017 of Lecture Notes in Mathematics, Springer, Heidelberg, Germany, 2011.

[16] C. B. Morrey, Multiple Integrals in the Calculus of Variations, Springer, 1966.

[17] I. Ekeland and R. T'emam, Convex Analysis and Variational Problems, North-Holland, 1976.

[18] F. C. Liu, "A Luzin type property of Sobolev functions," Indiana University Mathematics Journal, vol. 26, no. 4, pp. 645-651, 1977.

[19] E. Acerbi and N. Fusco, "Semicontinuity problems in the calculus of variations," Archive for Rational Mechanics and Analysis, vol. 86, no. 2, pp. 125-145, 1984.

[20] B. Dacorogna, Weak Continuity and Weak Lower Semicontinuity of Nonlinear Functionals, vol. 922 of Lecture Notes in Mathematics, Springer, Berlin, Germany, 1982.

[21] D. Gilbarg and N. S. Trudinger, Elliptic Partial Differential Equations of Second Order, Springer, 1998. 


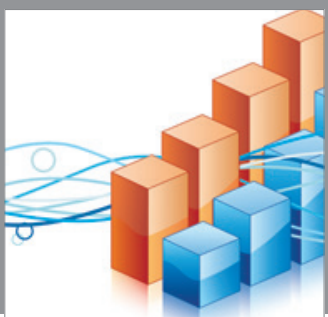

Advances in

Operations Research

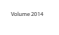

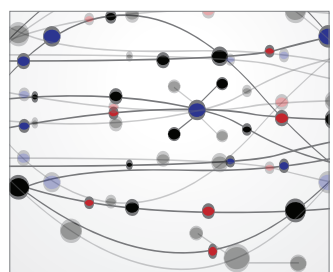

\section{The Scientific} World Journal
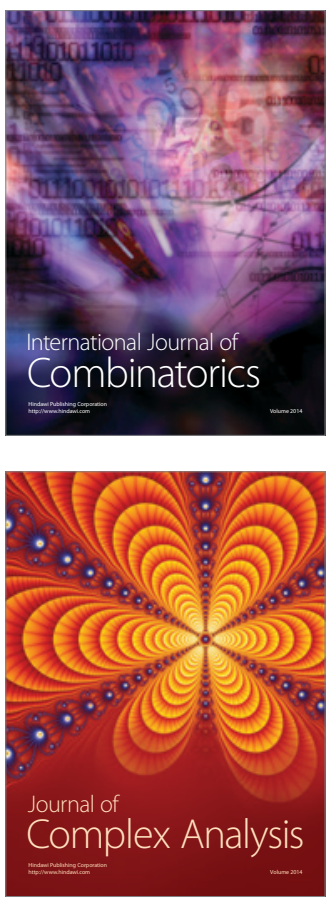

International Journal of

Mathematics and

Mathematical

Sciences
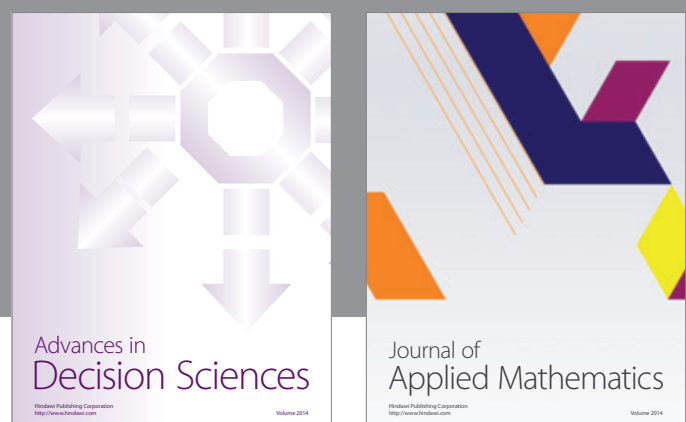

Journal of

Applied Mathematics
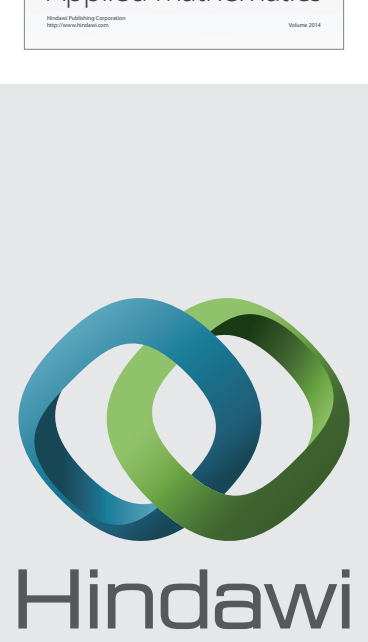

Submit your manuscripts at http://www.hindawi.com
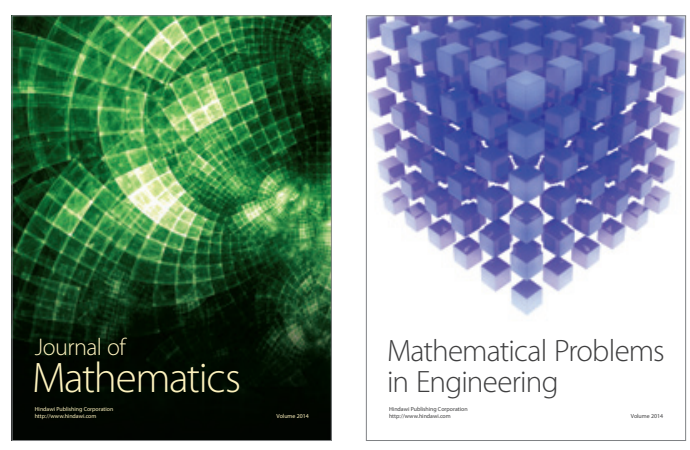

Mathematical Problems in Engineering
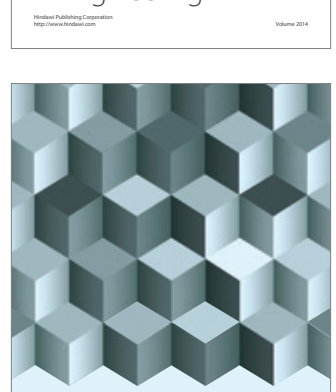

Journal of

Function Spaces
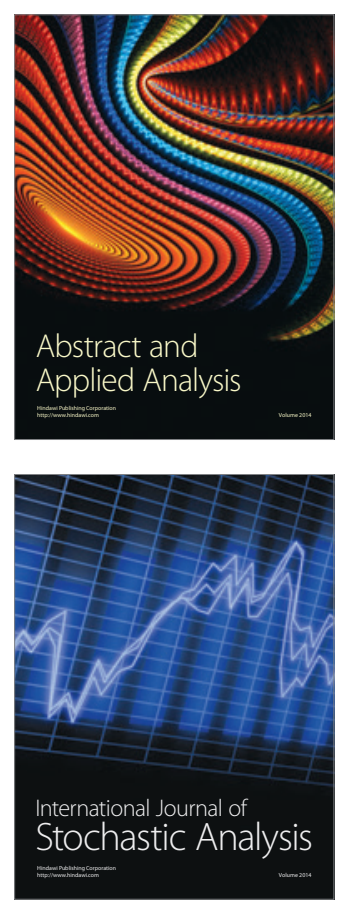

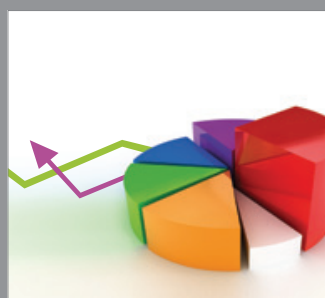

ournal of

Probability and Statistics

Promensencen
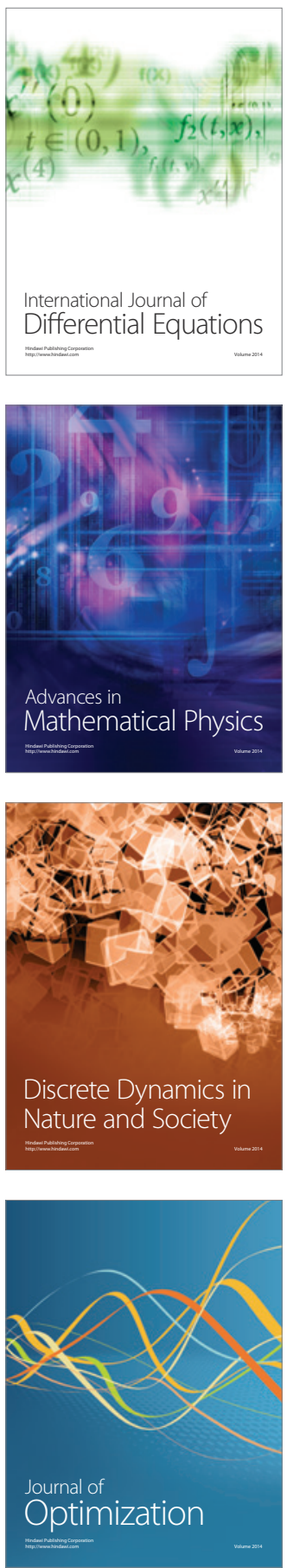